\title{
Does the Combination of Resistance Training and A Nutritional Intervention Have A Synergic Effect on Muscle Mass, Strength, and Physical Function in Older Adults? A Systematic Review and Meta-Analysis
}

\author{
MoonKi Choi \\ Kangwon National University \\ Hayeon Kim \\ Seoul Women's College of Nursing \\ Juyeon Bae ( $\square$ porester@naver.com) \\ Yeoju Institute of Technology
}

\section{Research Article}

Keywords: exercise, frailty, nutrition, older adults, resistance training, sarcopenia

Posted Date: July 16th, 2021

DOI: https://doi.org/10.21203/rs.3.rs-596096/v1

License: (ㄷ) (i) This work is licensed under a Creative Commons Attribution 4.0 International License. Read Full License

Version of Record: A version of this preprint was published at BMC Geriatrics on November 12th, 2021. See the published version at https://doi.org/10.1186/s12877-021-02491-5. 


\section{Abstract}

Background: Health-promoting interventions are important to prevent frailty and sarcopenia in older adults. However, there is limited evidence that nutritional interventions yield additional effects when combined with resistance training. This systematic review and meta-analysis aimed to compare the effectiveness of nutritional interventions with resistance training and that of resistance training only.

Methods: Randomized controlled trials published in peer-reviewed journals prior to July 2020 were retrieved from databases and other sources. The articles were screened according to the inclusion and exclusion criteria. The methodological quality of the included studies was assessed using Cochrane's risk of bias tool 2. A meta-analysis was performed using the RevMan 5.4 program.

Results: A total of 26 studies were included in the meta-analysis. The results of the meta-analysis showed no significant differences between groups in lean body mass, appendicular skeletal muscle mass, hand grip strength, knee extension, chair stand test results, or the timed up-and-go test results. In the subgroup analysis regarding the types of nutritional interventions, creatine showed significant effects on lean body mass ( $n=3, \mathrm{MD} 2.96, \mathrm{Cl} 0.76$ to 5.16 ). Regarding the other subgroup analyses, there were no significant differences in the mean age or sex of the participants, type of nutritional intervention, or duration of intervention.

Conclusions: This meta-analysis showed that the addition of nutritional interventions to resistance training has no additional effect on body composition, muscle strength, or physical function. Only creatine showed synergistic effects with resistance training on muscle mass.

\section{Trial Registration}

CRD42021224843

\section{Background}

Age-related conditions and chronic diseases increase the risk of disability and dependence, which are considered nearly irreversible conditions. Increasingly more older adults are becoming interested in 'active aging', which refers to the process of optimizing opportunities for health, participation, and security later in life [1]. A growing research interest is the identification of factors that increase the risk of negative events and the development of preventive interventions against disability. In this context, frailty and sarcopenia have increasingly emerged as research interests.

Although there is still no consensus on the definition and measurement of frailty for diagnosis, frailty is defined as a geriatric condition characterized by a cumulative decline in functioning and accompanied by increased vulnerability to stressors and dependency [2]. In 2001, Fried et al. [3] suggested the following criteria of frailty as a physical phenotype, focusing on physiological components: unintentional weight loss, exhaustion, decreased physical activity, a slow walking speed, and muscle weakness. Rockwood and Mitnitski [4] introduced a frailty index based on the accumulation of age-related deficits. A recent consensus suggested more broadly that frailty is a multidimensional syndrome including sensory limitations, cognitive decline, moodrelated conditions, changes in the social environment, comorbidities and disability in addition to physical impairment [5]. The specific pathological pathway of frailty remains unclear, but frailty has a biological component resulting from inflammation and cumulative cellular damage over one's lifetime. Although it occurs independently of chronological age, frailty is more prevalent in people of an older age; females; those who are living alone; those with low educational and socioeconomic statuses, multimorbidity, malnourishment, depression, polypharmacy, cognitive impairment, and a low physical activity level; and those who smoke and drink alcohol regularly [6-8].

Sarcopenia is considered a muscle disorder associated with poor muscle function; low muscle mass is considered a principal determinant. Although sarcopenia occurs in people who are not elderly, muscle mass decreases with age [9]. There are several operational definitions of sarcopenia; for example, the European working group on sarcopenia in older people defines sarcopenia as a combination of low muscle mass and strength and/or poor physical function [10]. Inconsistency in the definition leads to a wide range of prevalence rates, ranging from 9.9-40.4\% [11]. Although the concepts of both frailty and sarcopenia are still being developed, the physical phenotypes of frailty, including low grip strength and slow gait speed described by Fried et al. [3] overlap substantially with those of sarcopenia [12]. In addition, as the etiology of frailty, such as inflammation, cellular damage, and protein degradation, is also related to that of sarcopenia, as sarcopenia is an essential component of physical frailty. Frailty with sarcopenia can result in falls and fractures, a loss of independence, disability, morbidities, social isolation, institutionalization, and hospitalization [6, 13, 14], which lead to increases in healthcare costs and social burden [15]. Physical frailty and sarcopenia are transitional processes that increase individuals' vulnerability to reduced functional capacity and adverse health outcomes. Issues related to healthcare and support for frail and sarcopenic older adults are expected to increase with population aging [16].

Health-promoting behaviors are important to prevent disability and dependence and to reduce the need for care [17]. Physical inactivity and malnutrition are common conditions in older adults and are major modifiable risk factors for frailty and sarcopenia [18, 19]. An increasing amount of research has suggested that physical inactivity can lead to the loss of muscle mass, decreases in muscle strength and poor physical performance. Several evidencebased systematic reviews and meta-analyses of RCTs have shown that exercise affects muscle mass, strength, and physical performance [17, 20]. For optimal effects, multimodal exercise combined with moderate- to high-intensity progressive resistance training and functional balance and mobility training at least twice a week for $30-45$ minutes per session is recommended [19, 21].

Several nutrients, such as protein and vitamins D and E, have been known to affect anabolic stimuli, lead to the synthesis of muscle proteins, and protect against oxidative damage and the loss of muscle mass [22]. Although nutrition plays a key role in the pathogenesis of physical frailty and sarcopenia, the 
effects of nutritional interventions on muscle mass, strength and physical function are unclear. A systematic review showed that exercise training, when combined with dietary supplementation, has been shown to yield additional effects on muscle mass, strength and physical performance in some studies, but the existing evidence was inconsistent [23]. A more recent systematic review and meta-analysis by Hita-Contreras et al. showed that nutritional interventions do not provide additional or synergistic benefits when combined with resistance exercise in terms of muscle strength and mobility improvements among older adults with sarcopenic obesity [20].

There is evidence suggesting that there is an interaction effect between exercise and various nutritional factors, particularly protein and some multinutrient supplements, that can slow age-related decline and preserve muscle function in older adults. However, whether this effect is a meaningful preventive effect on frailty and sarcopenia remains unclear. Some previous reviews did not provide a quantitative synthesis, combined community-dwelling and institutionalized populations, or included and analyzed diverse types of interventions together [17, 23, 24], making it difficult to interpret the results. Thus, we focused on the primary prevention and synergistic effects of nutritional interventions, that is, the changes in muscle function after resistance training and nutritional interventions, in healthy community-dwelling older adults. The aim of this systematic review and meta-analysis was to compare the combination of resistance training and nutritional interventions with resistance training only. A PRISMA checklist is represented in Additional file 1.

\section{Methods}

\section{Search Strategy}

Electronic databases and the reference lists of related studies were searched by two investigators. First, for the electronic search, MEDLINE (PubMed), Cochrane CENTRAL, and EMBASE were searched for articles published prior to July 2020 by entering the following combinations of keywords: ("nutrition" OR "food" OR "diet") OR ("exercise" OR "resistance training") AND "aged" AND ("muscle mass" or "skeletal muscle" OR "muscle strength" OR "physical performance" OR "physical functional performance" OR "walking speed" OR "gait speed"). Second, the reference lists of related studies were searched to identify additional articles. The searches were limited to articles published in the English language, studies involving humans, and RCTs. Only peerreviewed articles were included, and grey literature such as dissertations, proceedings, and government reports was excluded.

\section{Study Selection}

The inclusion criteria for this systematic review were as follows: (a) studies including community-dwelling healthy older adults aged 60 or above; (b) those including experimental groups that underwent resistance training and nutritional interventions; (c) those including comparison groups that underwent resistance training only with or without a placebo supplement; (d) studies that reported the outcome measures of muscle mass, muscle strength, and physical functional performance; and (e) randomized controlled parallel-group trials with at least one arm. We included only studies in healthy subjects to reduce the level of heterogeneity between studies. We accepted the various authors' own definitions of 'healthy'. The experimental interventions included any form of resistance training and nutritional (dietary) interventions that involved repeated practice during standardized programs for the purpose of enhancing muscle mass, muscle strength, and physical function. Resistance training included multimodal exercises, including aerobic and balance exercises along with resistance exercises as well as resistance exercises only. Nutritional interventions were defined as those that provided at least one nutrient through nutritional supplementation or whole food to obtain biologically beneficial effects. There was no minimum duration of follow-up. However, all included trials had to report outcomes at a minimum of one time point after the completion of the intervention.

Articles were excluded if (a) the participants had malignant tumors, severe chronic diseases, or levels of frailty and sarcopenia that limited their physical activity, diet, and level of independence in daily life; (b) the study was conducted in an animal model; (c) the experimental intervention was combined with any other form of interventions such as medication and hormone therapy; (d) the nutritional intervention was designed for calorie intake reduction and weight loss; (e) the study evaluated the effectiveness of experimental interventions by only examining inflammatory factors or biological markers related to muscle synthesis, or (f) the study had a non-RCT design such as case reports or cohort studies without a comparison group.

Studies were selected based on the inclusion and exclusion criteria by two independent researchers; these researchers screened the studies according to the titles and abstracts of all studies and then reviewed full texts of the remaining studies. Disagreements between researchers were resolved by discussion.

\section{Data Extraction}

Two independent researchers extracted key data from the included articles in a standardized Excel sheet, and the results were cross checked. For each article, data about (a) the article, including the authors, year of publication, and country; (b) characteristics of the study population, including the number of participants, mean age, sex, health status, and attrition rate; (c) characteristics of the experimental intervention, including the contents of resistance training, contents of nutritional intervention, delivery mode, amount, frequency and duration of intervention, and treatment for comparison group; and (d) outcome evaluation, including the follow-up period, method of measurement, and all outcome measured. As the aim of the study was to compare the effects of the combination of resistance training and nutritional interventions with those of resistance training only on muscle mass, strength, and physical performance, when more than two groups were present, only the data regarding the two groups we intended to compare were recorded.

\section{Assessment of Study Quality (Assessment of Risk of Bias)}

Methodological quality was assessed using Cochrane's risk of bias 2 (RoB2) tool by two independent researchers. The RoB2 tool consists of five domains: the randomization process, deviation from intended intervention, missing outcome data, measurement of outcome, and selection of the reported result. The risk of bias for each domain is evaluated as a "low risk", "some concerns", or a "high risk" by an algorithm with several signaling questions. Overall, 
"low risk of bias" was recorded when the study was judged to have a low risk of bias for all domains, "some concerns" was recorded when the study was judged to have some concerns in at least one domain, and "high risk of bias" was recorded when the study was judged to have a high risk of bias in at least one domain. This process was carried out by two independent researchers, and inconsistencies on items were resolved through discussion.

\section{Data Synthesis and Statistical Analysis}

The effect sizes of the combination of resistance training and a nutritional intervention were calculated using the mean difference (MD) or standardized MD (SMD) for continuous outcome data for muscle mass, muscle strength, and physical functional performance. When a study provided data on more than one outcome for the same construct (ex: timed up-and-go and $4 \mathrm{~m}$ walk tests for physical functional performance), the valid, reliable and commonly used measures for frailty and sarcopenia were selected by reviewing the associated literature and considering the frequencies of their use in the included studies. As a result, lean body mass and appendicular skeletal muscle mass were selected for muscle mass, grip strength and knee extension for muscle strength, and the chair stand and timed up-and-go tests for physical functional performance. Fat-free mass was included in the analysis when lean body mass was not available. Both isometric strength and isotonic strength in knee extension were included, and when both of them were measured in the same study, isotonic strength was selected.

In addition, if a study used different lengths of intervention and follow-up periods, we used the outcome values at the postintervention endpoint. When only the mean change scores and standard deviation (SD) of each group were available, they were used instead of the postintervention endpoint mean and SD for the mean difference. SMDs were used for studies using different units (scale) of the same measure (ex: $\mathrm{kg}$ and $\mathrm{Nm}$ for strength). If there were more than two groups that could be considered experimental groups in the study, the group corresponding to the treatment that was expected to be more effective under the hypothesis or the group with a higher intensity of intervention was included as an experimental group in the meta-analysis. Studies for which we could not identify the outcome data necessary for quantitative synthesis after contacting the authors were excluded from this meta-analysis

Individual MDs and SMDs were pooled using random-effects models and the inverse variance method. The statistical significance of each effect size and overall effect size were checked using 95\% confidence intervals. The chi-squared test and Higgin's $\mathrm{I}^{2}$ test were used to examine between-trial heterogeneity. When the $p$ value for the chi-squared test was less than 0.1 and $\mathrm{I}^{2}$ was greater than $50 \%$, heterogeneity was considered present. Subgroup analysis was conducted by the nutritional intervention type (creatine, multinutrients, protein, and vitamin $\mathrm{D}$ ), duration of intervention (< 16 weeks and $\geq 16$ weeks), and participant type ( $<70$ years and $\geq 70$ years; female, male, and mixed). All subgroup differences were tested regarding the significance of the effect sizes and heterogeneity. All analyses were conducted using Review Manager (RevMan) 5.4.

\section{Results}

\section{Search Results}

Figure 1 demonstrates the study selection process. After duplicates were removed, 3,638 articles remained. After 3,549 articles were excluded through title and abstract review, the full texts of 89 articles were reviewed. Sixty-two articles were additionally excluded, and consequently, 27 articles (26 RCTs in 27 papers) were included in this systematic review; among the 27 articles, two articles reported information from the same RCT [25, 26]. In some papers, the necessary values for meta-analysis could not be identified, so 26 articles were included in the quantitative synthesis.

\section{Description of Included Studies}

Table 1 shows the characteristics of the included studies. Six RCTs were conducted in Canada, six in Japan, three in Brazil, three in the UK, two in the Netherlands, and one each in Australia, Chile, Iceland, Norway, Sweden, and the USA; the studies were published between 2001 and 2020. The sample sizes ranged from 18 to 161 . Six studies were conducted in males only, five studies were conducted in females only, and 15 studies were conducted in both males and females. There were 12 studies with a mean age of participants of less than 70 years and 14 studies with a mean age of more than 70 years. 
Table 1

Summary of included study characteristics

\begin{tabular}{|c|c|c|c|c|c|c|c|c|}
\hline \multirow{2}{*}{$\begin{array}{l}\text { First author } \\
\text { (year), study } \\
\text { location }\end{array}$} & \multirow{2}{*}{$\begin{array}{l}\text { Sample } \\
\text { characteristics: } \\
\text { n, mean age } \pm \\
\text { SD, sex } \\
\text { (female ratio) }\end{array}$} & \multicolumn{2}{|c|}{ Intervention group } & \multirow{2}{*}{$\begin{array}{l}\text { Control } \\
\text { groupt }\end{array}$} & \multirow{2}{*}{$\begin{array}{l}\text { Follow- } \\
\text { up } \\
\text { period } \\
\text { (weeks) }\end{array}$} & \multirow{2}{*}{$\begin{array}{l}\text { Body } \\
\text { composition } \\
\text { assessment } \\
\text { method }\end{array}$} & \multirow{2}{*}{$\begin{array}{l}\text { Muscle } \\
\text { strength } \\
\text { assessment } \\
\text { method }\end{array}$} & \multirow{2}{*}{$\begin{array}{l}\text { Physical } \\
\text { performance } \\
\text { assessment } \\
\text { method }\end{array}$} \\
\hline & & Exercise & Nutrition & & & & & \\
\hline \multirow{3}{*}{$\begin{array}{l}\text { Aguiar } \\
\text { (2013), } \\
\text { Brazil }\end{array}$} & \multirow{3}{*}{$\begin{array}{l}\text { I: } 9,64 \pm 4, \\
\text { female } \\
\text { C: } 9,65 \pm 6 \\
\text { female }\end{array}$} & \multirow{3}{*}{$\begin{array}{l}\text { Resistance } \\
\text { training } \\
\text { using } \\
\text { machines, } \\
60 \text { min } \\
\text { sessions, } 3 \\
\text { times a } \\
\text { week, for } 24 \\
\text { weeks }\end{array}$} & \multirow{3}{*}{$\begin{array}{l}5 \mathrm{~g} \text { Creatine } \\
\text { monohydrate, once a } \\
\text { day, for } 12 \text { weeks }\end{array}$} & \multirow[t]{3}{*}{$\begin{array}{l}\text { Placebo: } \\
\text { maltodextrin }\end{array}$} & \multirow[t]{3}{*}{24} & \multirow{2}{*}{$\begin{array}{l}\text { Body mass } \\
\text { Fat free } \\
\text { mass }\end{array}$} & \multirow{2}{*}{$\begin{array}{l}\text { Bench } \\
\text { press } \\
\text { Biceps curl }\end{array}$} & \multirow[t]{3}{*}{ Chair stand } \\
\hline & & & & & & & & \\
\hline & & & & & & $\begin{array}{l}\text { Muscle } \\
\text { mass }\end{array}$ & $\begin{array}{l}\text { Knee } \\
\text { extension }\end{array}$ & \\
\hline \multirow{4}{*}{$\begin{array}{l}\text { Aoki (2018), } \\
\text { Japan }\end{array}$} & \multirow{2}{*}{$\begin{array}{l}\text { I: } 43,68.8 \pm \\
5.3, \text { mixed } \\
(74.4 \%)\end{array}$} & \multirow{4}{*}{$\begin{array}{l}\text { Lower body } \\
\text { resistance } \\
\text { training, } \\
\text { daily }\end{array}$} & \multirow{4}{*}{$\begin{array}{l}25 \mathrm{mcg} \text { vitamin D3 } \\
(1000 \text { IU), divided into } 3 \\
\text { times, daily }\end{array}$} & \multirow[t]{4}{*}{ Usual diet } & \multirow[t]{4}{*}{24} & & Hip flexion & Chair stand \\
\hline & & & & & & $\begin{array}{l}\text { Lower limb } \\
\text { muscle }\end{array}$ & $\begin{array}{l}\text { Knee } \\
\text { extension }\end{array}$ & $\begin{array}{l}\text { Single leg } \\
\text { stance }\end{array}$ \\
\hline & \multirow[t]{2}{*}{$\begin{array}{l}\text { C: } 45,71.2 \pm \\
6.8, \text { mixed } \\
(75.6 \%)\end{array}$} & & & & & mass & & $\begin{array}{l}\text { Two step } \\
\text { test }\end{array}$ \\
\hline & & & & & & & & $\begin{array}{l}\text { Functional } \\
\text { reach test }\end{array}$ \\
\hline \multirow{3}{*}{$\begin{array}{l}\text { Arnarson } \\
\text { (2013), } \\
\text { Iceland }\end{array}$} & \multirow{3}{*}{$\begin{array}{l}\text { I: } 83,73.3 \pm 6 \text {, } \\
\text { mixed } \\
\text { (unknown) } \\
\text { C: } 78,74.6 \pm \\
5.8, \text { mixed } \\
\text { (unknown) }\end{array}$} & \multirow{3}{*}{$\begin{array}{l}\text { Resistance } \\
\text { training } \\
\text { using } \\
\text { machines, } 3 \\
\text { times a } \\
\text { week }\end{array}$} & \multirow{3}{*}{$\begin{array}{l}20 \mathrm{~g} \text { of whey protein } 3 \\
\text { times a week }\end{array}$} & \multirow{3}{*}{$\begin{array}{l}\text { Placebo: } \\
\text { 250ml } \\
\text { isocaloric } \\
\text { carbohydrate } \\
\text { drink }\end{array}$} & \multirow[t]{3}{*}{12} & & $\begin{array}{l}\text { Grip } \\
\text { strenath }\end{array}$ & TUG \\
\hline & & & & & & $\begin{array}{l}\text { Lean body } \\
\text { mass }\end{array}$ & Knee & 6-min walk \\
\hline & & & & & & & extension & \\
\hline $\begin{array}{l}\text { Bjørnsen } \\
\text { (2016), }\end{array}$ & $\begin{array}{l}\text { I: } 17,69 \pm 7 \\
\text { male }\end{array}$ & $\begin{array}{l}\text { Free weight } \\
\text { exercises, } 3\end{array}$ & $\begin{array}{l}500 \mathrm{mg} \text { vitamin } \mathrm{C} \text { and } \\
117.5 \mathrm{mg} \text { vitamin } \mathrm{E}\end{array}$ & $\begin{array}{l}\text { Placebo: } \\
\text { cellulose and }\end{array}$ & 12 & $\begin{array}{l}\text { Lean body } \\
\text { mass }\end{array}$ & & - \\
\hline Norway & $\begin{array}{l}\text { C: } 17,67 \pm 5 \\
\text { male }\end{array}$ & $\begin{array}{l}\text { times a } \\
\text { week }\end{array}$ & twice a day & $\begin{array}{l}\text { dicalsium } \\
\text { phosphate }\end{array}$ & & $\begin{array}{l}\text { Muscle } \\
\text { thickness }\end{array}$ & $\begin{array}{l}\text { Knee } \\
\text { extension }\end{array}$ & \\
\hline & & & & & & & Leg press & \\
\hline Bobeuf & I: $14,64.3 \pm$ & Resistance & 1000mg vitamin C & Placebo: & 24 & ASMM & - & - \\
\hline Canada & $(50 \%)$ & min & $\mathrm{E}$, once a day & lactose & & Muscle & & \\
\hline & $\begin{array}{l}\text { C: } 17,67 \pm 3.7 \\
\text { mixed }(52.9 \%)\end{array}$ & $\begin{array}{l}\text { times a } \\
\text { week }\end{array}$ & & & & & & \\
\hline $\begin{array}{l}\text { Brose(2003), } \\
\text { Canada }\end{array}$ & $\begin{array}{l}\text { I: } 14,69.6 \pm \\
5.4, \text { mixed } \\
(42.8 \%)\end{array}$ & $\begin{array}{l}\text { Resistance } \\
\text { training } \\
\text { using }\end{array}$ & $\begin{array}{l}5 \mathrm{~g} \text { creatine and } 2 \mathrm{~g} \\
\text { dextrose, once a day }\end{array}$ & $\begin{array}{l}\text { Placebo: } \\
\text { 7g dextrose }\end{array}$ & 14 & $\begin{array}{l}\text { Fat free } \\
\text { mass }\end{array}$ & $\begin{array}{l}\text { Grip } \\
\text { strength }\end{array}$ & - \\
\hline & C: $14,69.1 \pm$ & $\begin{array}{l}\text { machines, } 3 \\
\text { times a }\end{array}$ & & & & & $\begin{array}{l}\text { Knee } \\
\text { extension }\end{array}$ & \\
\hline & $(50 \%)$ & week & & & & & Leg press & \\
\hline $\begin{array}{l}\text { Bunout } \\
\text { (2006), Chile }\end{array}$ & $\begin{array}{l}\text { I: } 24,78 \pm 4 \\
\text { mixed }(91.6 \%)\end{array}$ & $\begin{array}{l}\text { Multimodal } \\
\text { exercise: }\end{array}$ & $\begin{array}{l}800 \mathrm{mg} \text { calcium and } \\
\text { vitamin D3 } 400 \mathrm{IU} \text {, once }\end{array}$ & $\begin{array}{l}800 \mathrm{mg} \\
\text { calcium }\end{array}$ & 36 & - & $\begin{array}{l}\text { Grip } \\
\text { strength }\end{array}$ & Body sway \\
\hline & $\begin{array}{l}\text { C: } 24,76 \pm 4 \\
\text { mixed }(87.5 \%)\end{array}$ & $\begin{array}{l}\text { balance and } \\
\text { aerobic }\end{array}$ & & & & & $\begin{array}{l}\text { Knee } \\
\text { extension }\end{array}$ & ratio \\
\hline & & training, & & & & & & SPPB \\
\hline & & 90 min & & & & & & TUG \\
\hline & & biweekly & & & & & & 12-min walk \\
\hline $\begin{array}{l}\text { Chrusch } \\
\text { (2001), } \\
\text { Canada }\end{array}$ & $\begin{array}{l}\mathrm{I}: 16,70.4 \pm 6.4 \\
\text { male }\end{array}$ & $\begin{array}{l}\text { Resistance } \\
\text { training }\end{array}$ & $\begin{array}{l}\text { Creatine supplement: } \\
0.3 \mathrm{~g} / \mathrm{kg} / \text { day for the first } \\
5 \text { davs(loading }\end{array}$ & $\begin{array}{l}\text { Placebo: } \\
\text { sucrose-flour }\end{array}$ & 12 & $\begin{array}{l}\text { Lean body } \\
\text { mass }\end{array}$ & $\begin{array}{l}\text { Bench } \\
\text { press }\end{array}$ & - \\
\hline & $\begin{array}{l}\text { C: } 14,71.1 \pm \\
6.7, \text { male }\end{array}$ & $\begin{array}{l}\text { machines, } 3 \\
\text { times a } \\
\text { week }\end{array}$ & $\begin{array}{l}\text { and } 0.07 \mathrm{~g} / \mathrm{kg} / \mathrm{day} \\
\text { thereafter, once a day, } \\
\text { for } 11 \text { weeks }\end{array}$ & & & & $\begin{array}{l}\text { Knee } \\
\text { extension }\end{array}$ & \\
\hline & & & & & & & Leg press & \\
\hline
\end{tabular}

TThe resistance exercise of the control group applied in the same manner as in the experimental group.

† The duration of intervention in most studies were the same as the follow-up period.

‡ ASMM: appendicular skeletal muscle mass, BMI : body mass index, C: control group, DHA: docosahexaenoic acid, EPA: eicosapentaenoic acid, I: intervention group, SMl; skeletal muscle massi index, SPPB: short physical performance battery, TUG: time up and go 


\begin{tabular}{|c|c|c|c|c|c|c|c|c|}
\hline \multirow{2}{*}{$\begin{array}{l}\text { First author } \\
\text { (year), study } \\
\text { location }\end{array}$} & \multirow{2}{*}{$\begin{array}{l}\text { Sample } \\
\text { characteristics: } \\
\text { n, mean age } \pm \\
\text { SD, sex } \\
\text { (female ratio) }\end{array}$} & \multicolumn{2}{|c|}{ Intervention group } & \multirow{2}{*}{$\begin{array}{l}\text { Control } \\
\text { groupt }\end{array}$} & \multirow{2}{*}{$\begin{array}{l}\text { Follow- } \\
\text { up } \\
\text { period } \\
\text { (weeks) }\end{array}$} & \multirow{2}{*}{$\begin{array}{l}\text { Body } \\
\text { composition } \\
\text { assessment } \\
\text { method }\end{array}$} & \multirow{2}{*}{$\begin{array}{l}\text { Muscle } \\
\text { strength } \\
\text { assessment } \\
\text { method }\end{array}$} & \multirow{2}{*}{$\begin{array}{l}\text { Physical } \\
\text { performance } \\
\text { assessment } \\
\text { method }\end{array}$} \\
\hline & & Exercise & Nutrition & & & & & \\
\hline \multirow[t]{3}{*}{$\begin{array}{l}\text { Cornish } \\
\text { (2018), } \\
\text { Canada }\end{array}$} & $\begin{array}{l}\mathrm{l}: 11,71.4 \pm 6.2 \\
\text { male } \\
\text { C: } 12,70.9 \pm 5 \\
\text { male }\end{array}$ & \multirow[t]{3}{*}{$\begin{array}{l}\text { Resistance } \\
\text { training, } 60 \\
\text { min } \\
\text { sessions, } 3 \\
\text { times a } \\
\text { week }\end{array}$} & \multirow{3}{*}{$\begin{array}{l}\text { combined } 1.98 \mathrm{~g} \text { EPA } \\
\text { and } 0.99 \mathrm{~g} \mathrm{DHA} \text {, once a }\end{array}$} & Placebo: & \multirow[t]{3}{*}{12} & \multirow{3}{*}{$\begin{array}{l}\text { Lean body } \\
\text { mass }\end{array}$} & \multirow[t]{3}{*}{ Chest press } & \multirow{3}{*}{ 6-min walk } \\
\hline & & & & $\begin{array}{l}3.0 \mathrm{~g} \text { omega } \\
3-6-9 \text { blend }\end{array}$ & & & & \\
\hline & & & & & & & & \\
\hline \multirow{2}{*}{$\begin{array}{l}\text { Da Boit } \\
\text { (2017), UK }\end{array}$} & $\begin{array}{l}\text { I: } 27,70.1 \pm 4 \\
\text { mixed }(48.1 \%)\end{array}$ & \multirow{2}{*}{$\begin{array}{l}\text { Lower body } \\
\text { resistance } \\
\text { training, } \\
\text { twice a } \\
\text { week }\end{array}$} & \multirow{2}{*}{$\begin{array}{l}3.0 \mathrm{~g} \text { omega }-3 \text { fatty } \\
\text { acids containing } 2.1 \mathrm{~g} \\
\text { EPA and } 0.6 \mathrm{~g} \text { DHA, } \\
\text { once } a \text { day }\end{array}$} & \multirow{2}{*}{$\begin{array}{l}\text { Placebo: } \\
3.0 \mathrm{~g} \\
\text { safflower oil }\end{array}$} & \multirow[t]{2}{*}{18} & \multirow{2}{*}{$\begin{array}{l}\text { Muscle } \\
\text { anatomic } \\
\text { cross- } \\
\text { sectional } \\
\text { area }\end{array}$} & \multirow{2}{*}{$\begin{array}{l}\text { Knee } \\
\text { extension }\end{array}$} & Chair stand \\
\hline & $\begin{array}{l}\text { C: } 23,70.9 \pm \\
4.2, \text { mixed } \\
(43.4 \%)\end{array}$ & & & & & & & $4 \mathrm{~m}$ walk \\
\hline \multirow[t]{5}{*}{$\begin{array}{l}\text { Dulac(2020), } \\
\text { UK }\end{array}$} & $\begin{array}{l}\text { I: } 21,68.3 \pm \\
5.3, \text { male }\end{array}$ & \multirow{5}{*}{$\begin{array}{l}\text { Resistance } \\
\text { training with } \\
\text { functional } \\
\text { exercises, } \\
60 \text { min } \\
\text { sessions, } 3 \\
\text { times a } \\
\text { week }\end{array}$} & \multirow{5}{*}{$\begin{array}{l}\text { Fast-whey protein: } \\
10 \mathrm{~g} \text { milk proteins, } 3 \\
\text { times a day }\end{array}$} & \multirow{5}{*}{$\begin{array}{l}\text { Placebo: } \\
\text { isocaloric } \\
\text { maltodextrin }\end{array}$} & \multirow[t]{5}{*}{12} & \multirow{5}{*}{$\begin{array}{l}\text { Lean body } \\
\text { mass } \\
\text { Fat mass } \\
\text { Lower limb } \\
\text { lean mass }\end{array}$} & \multirow[t]{5}{*}{$\begin{array}{l}\text { Grip } \\
\text { strength }\end{array}$} & Chair stand \\
\hline & C: $19,70.7 \pm$ & & & & & & & Stalr cllmb \\
\hline & 8.0, male & & & & & & & balance \\
\hline & & & & & & & & TUG \\
\hline & & & & & & & & $4 \mathrm{~m}$ walk \\
\hline \multirow{2}{*}{$\begin{array}{l}\text { Edholom } \\
\text { (2017), } \\
\text { Sweden }\end{array}$} & $\begin{array}{l}\text { I: } 20,67.2 \pm \\
1.3, \text { female }\end{array}$ & \multirow{2}{*}{$\begin{array}{l}\text { Resistance } \\
\text { training, } 60 \\
\text { min } \\
\text { sessions, } \\
\text { twice a } \\
\text { week }\end{array}$} & \multirow{2}{*}{$\begin{array}{l}\text { Healthy diet : } \\
\text { following a dietary } \\
\text { consultation and a diet } \\
\text { plan with the current } \\
\text { dietary guidelines in } \\
\text { Europe and US }\end{array}$} & \multirow[t]{2}{*}{ Usual diet } & \multirow[t]{2}{*}{24} & \multirow[t]{2}{*}{$\begin{array}{l}\text { Lean body } \\
\text { mass }\end{array}$} & $\begin{array}{l}\text { Knee } \\
\text { extension }\end{array}$ & Chair stand \\
\hline & $\begin{array}{l}\text { C: } 17,67.9 \pm \\
2.1, \text { female }\end{array}$ & & & & & & Leg press & $\begin{array}{l}\text { stance } \\
\text { Squat jump } \\
\text { TUG }\end{array}$ \\
\hline $\begin{array}{l}\text { Formica } \\
(2020)\end{array}$ & $\begin{array}{l}\text { I: } 77,71.2 \pm \\
4.0, \text { mixed }\end{array}$ & $\begin{array}{l}\text { Multimodal } \\
\text { exercise: }\end{array}$ & $\begin{array}{l}\sim 220 \mathrm{~g} \text { of lean red meat } \\
\text { or } 160 \mathrm{~g} \text { of cooked red }\end{array}$ & Usual diet & 24 & ASMM & $\begin{array}{l}\text { Knee } \\
\text { extension }\end{array}$ & Chair stand \\
\hline Austrália & $(62 \%)$ & $\begin{array}{l}\text { aerobic, } \\
\text { resistance, }\end{array}$ & $\begin{array}{l}\text { meat, twice a day- } \\
\text { across } 2 \text { meals, } 3 \text { times }\end{array}$ & & & $\begin{array}{l}\text { Lean body } \\
\text { mass }\end{array}$ & Leg press & TUG \\
\hline & $\begin{array}{l}\text { C: } 77,70.3 \pm \\
4.3, \text { mixed } \\
(62 \%)\end{array}$ & $\begin{array}{l}\text { balance and } \\
\text { mobility } \\
\text { training, } 60\end{array}$ & a week & & & $\begin{array}{l}\text { Lower limb } \\
\text { lean mass }\end{array}$ & & $\begin{array}{l}\text { 4m walk } \\
\text { 4-square }\end{array}$ \\
\hline & & $\begin{array}{l}\text { sessions, } 3 \\
\text { times a } \\
\text { week }\end{array}$ & & & & $\begin{array}{l}\text { Upper limb } \\
\text { lean mass }\end{array}$ & & \\
\hline
\end{tabular}

†The resistance exercise of the control group applied in the same manner as in the experimental group.

† The duration of intervention in most studies were the same as the follow-up period.

‡ ASMM: appendicular skeletal muscle mass, BMI : body mass index, C: control group, DHA: docosahexaenoic acid, EPA: eicosapentaenoic acid, I: intervention group, SMl; skeletal muscle massi index, SPPB: short physical performance battery, TUG: time up and go 


\begin{tabular}{|c|c|c|c|c|c|c|c|c|}
\hline \multirow{2}{*}{$\begin{array}{l}\text { First author } \\
\text { (year), study } \\
\text { location }\end{array}$} & \multirow{2}{*}{$\begin{array}{l}\text { Sample } \\
\text { characteristics: } \\
\text { n, mean age } \pm \\
\text { SD, sex } \\
\text { (female ratio) }\end{array}$} & \multicolumn{2}{|c|}{ Intervention group } & \multirow{2}{*}{$\begin{array}{l}\text { Control } \\
\text { groupt }\end{array}$} & \multirow{2}{*}{$\begin{array}{l}\text { Follow- } \\
\text { up } \\
\text { period } \\
\text { (weeks) }\end{array}$} & \multirow{2}{*}{$\begin{array}{l}\text { Body } \\
\text { composition } \\
\text { assessment } \\
\text { method }\end{array}$} & \multirow{2}{*}{$\begin{array}{l}\text { Muscle } \\
\text { strength } \\
\text { assessment } \\
\text { method }\end{array}$} & \multirow{2}{*}{$\begin{array}{l}\text { Physical } \\
\text { performance } \\
\text { assessment } \\
\text { method }\end{array}$} \\
\hline & & Exercise & Nutrition & & & & & \\
\hline $\begin{array}{l}\text { Holwerda } \\
\text { (2018), } \\
\text { Netherlands }\end{array}$ & $\begin{array}{l}\text { l: } 21,69 \pm 4.6 \text {, } \\
\text { male } \\
\text { C: } 20,71 \pm 4.5 \text {, } \\
\text { male }\end{array}$ & $\begin{array}{l}\text { Resistance } \\
\text { exercise } \\
\text { training, } \\
3 \text { times a } \\
\text { week }\end{array}$ & $\begin{array}{l}21 \mathrm{~g} \text { leucine-enriched } \\
\text { whey protein ( } 3 \mathrm{~g} \text { total } \\
\text { leucine), once a day }\end{array}$ & Placebo & 12 & $\begin{array}{l}\text { ASMM } \\
\text { BMI } \\
\text { Body mass } \\
\text { Fat mass } \\
\text { Lean body } \\
\text { mass }\end{array}$ & $\begin{array}{l}\text { Knee } \\
\text { extension } \\
\text { Leg press }\end{array}$ & $\begin{array}{l}\text { Chair stand } \\
\text { SPPB } \\
4 \mathrm{~m} \text { walk }\end{array}$ \\
\hline $\begin{array}{l}\text { Kawada } \\
\text { (2013), } \\
\text { Japan }\end{array}$ & $\begin{array}{l}\text { I: } 13,67 \pm 3 \\
\text { mixed }(61.5 \%) \\
\text { C: } 13,70 \pm 1 \\
\text { mixed }(53.8 \%)\end{array}$ & $\begin{array}{l}\text { Low- } \\
\text { intensity } \\
\text { resistance } \\
\text { training, } \\
\text { twice a } \\
\text { week }\end{array}$ & $\begin{array}{l}\text { 3.0g essential amino } \\
\text { acid supplements with } \\
\text { milk, twice a day }\end{array}$ & $\begin{array}{l}\text { Placebo: } \\
\text { 3g dextrin- } \\
\text { contained } \\
\text { powder with } \\
\text { milk, once a } \\
\text { day }\end{array}$ & 24 & $\begin{array}{l}\text { Cross } \\
\text { sectional } \\
\text { area of } \\
\text { Psoas } \\
\text { major } \\
\text { muscle }\end{array}$ & - & $\begin{array}{l}\text { Gait speed } \\
\text { Obstacle } \\
\text { course walk } \\
\text { 6-min walk }\end{array}$ \\
\hline $\begin{array}{l}\text { Kirk (2019), } \\
\text { UK }\end{array}$ & $\begin{array}{l}\text { I: } 22,69 \pm 6 \\
\text { mixed }(59.1 \%) \\
\text { C: } 24,66 \pm 4 \\
\text { mixed }(50 \%)\end{array}$ & $\begin{array}{l}\text { Resistance } \\
\text { exercise and } \\
\text { functional } \\
\text { exercise } \\
\text { with }\end{array}$ & $\begin{array}{l}\text { Whey protein } \\
(0.5 \mathrm{~g} / \mathrm{kg} / \mathrm{meal}) \text { mixed } \\
\text { with } \\
\text { leucine }(0.03 \mathrm{~g} / \mathrm{kg} / \mathrm{meal}) \text {, } \\
3 \text { times a day }\end{array}$ & Usual diet & 16 & BMI & $\begin{array}{l}\text { Leg press } \\
\text { Chest press } \\
\text { Biceps curl }\end{array}$ & $\begin{array}{l}\text { Obstacle } \\
\text { course walk } \\
\text { SPPB } \\
\text { 6-min walk }\end{array}$ \\
\hline $\begin{array}{l}\text { Kirk (2020), } \\
\text { UK }\end{array}$ & & $\begin{array}{l}\text { min } \\
\text { sessions, } \\
\text { twice a } \\
\text { week }\end{array}$ & & & & $\begin{array}{l}\text { Fat mass } \\
\text { Muscle } \\
\text { mass } \\
\text { SMI }\end{array}$ & $\begin{array}{l}\text { Grip } \\
\text { strength } \\
\text { Knee } \\
\text { extension } \\
\text { Knee } \\
\text { flexion }\end{array}$ & - \\
\hline $\begin{array}{l}\text { Leenders } \\
\text { (2013), } \\
\text { Netherlands }\end{array}$ & $\begin{array}{l}\text { I: } 27,70.9 \pm 5.4, \\
\text { mixed }(44.4 \%) \\
\text { C: } 26,69.5 \pm \\
3.6, \text { mixed } \\
(46.2 \%)\end{array}$ & $\begin{array}{l}\text { Resistance } \\
\text { training, } 3 \\
\text { times a } \\
\text { week }\end{array}$ & $\begin{array}{l}15 \mathrm{~g} \text { milk protein, once a } \\
\text { day }\end{array}$ & $\begin{array}{l}\text { Placebo: } \\
7.13 \mathrm{~g} \\
\text { lactose and } \\
0.42 \mathrm{~g} \\
\text { calcium only }\end{array}$ & 24 & $\begin{array}{l}\text { BMI } \\
\text { Lean body } \\
\text { mass } \\
\text { Lower limb } \\
\text { lean mass }\end{array}$ & $\begin{array}{l}\text { Grip } \\
\text { strength } \\
\text { Leg press }\end{array}$ & Chair stand \\
\hline $\begin{array}{l}\text { Mori (2018), } \\
\text { Japan }\end{array}$ & $\begin{array}{l}\text { I: } 25,70.6 \pm \\
4.2, \text { female } \\
\text { C: } 25,70.6 \pm \\
4.2, \text { female }\end{array}$ & $\begin{array}{l}\text { Resistance } \\
\text { training, } \\
\text { twice a } \\
\text { week }\end{array}$ & $\begin{array}{l}25 \mathrm{~g} \text { leucine enriched } \\
\text { whey protein, once a } \\
\text { day }\end{array}$ & Usual diet & 24 & $\begin{array}{l}\text { BMI } \\
\text { Lower limb } \\
\text { lean mass } \\
\text { SMI } \\
\text { Upper limb } \\
\text { lean mass }\end{array}$ & $\begin{array}{l}\text { Grip } \\
\text { strength } \\
\text { Knee } \\
\text { extension }\end{array}$ & Gait speed \\
\hline $\begin{array}{l}\text { Nabuco } \\
(2018) \\
\text { Brazil }\end{array}$ & $\begin{array}{l}\text { I: } 23,66.2 \pm \\
9.4 \text {, female } \\
\text { C: } 23,66.5 \pm \\
7.2 \text {, female }\end{array}$ & $\begin{array}{l}\text { Resistance } \\
\text { training, } 3 \\
\text { times a } \\
\text { week }\end{array}$ & $\begin{array}{l}27.1 \mathrm{~g} \text { whey protein, } 3 \\
\text { times a week }\end{array}$ & $\begin{array}{l}\text { Placebo: } \\
\text { maltodextrin } \\
\text { drink }\end{array}$ & 12 & $\begin{array}{l}\text { Lower limb } \\
\text { lean mass } \\
\text { Skeletal } \\
\text { muscle } \\
\text { mass } \\
\text { Upper limb } \\
\text { lean mass }\end{array}$ & $\begin{array}{l}\text { Biceps curl } \\
\text { Chest press } \\
\text { Knee } \\
\text { extension }\end{array}$ & $\begin{array}{l}\text { Chair stand } \\
\text { Gait speed }\end{array}$ \\
\hline
\end{tabular}

†The resistance exercise of the control group applied in the same manner as in the experimental group.

† The duration of intervention in most studies were the same as the follow-up period.

‡ ASMM: appendicular skeletal muscle mass, BMI : body mass index, C: control group, DHA: docosahexaenoic acid, EPA: eicosapentaenoic acid, I: intervention group, SMl; skeletal muscle massi index, SPPB: short physical performance battery, TUG: time up and go 


\begin{tabular}{|c|c|c|c|c|c|c|c|c|}
\hline \multirow{2}{*}{$\begin{array}{l}\text { First author } \\
\text { (year), study } \\
\text { location }\end{array}$} & \multirow{2}{*}{$\begin{array}{l}\text { Sample } \\
\text { characteristics: } \\
\text { n, mean age } \pm \\
\text { SD, sex } \\
\text { (female ratio) }\end{array}$} & \multicolumn{2}{|c|}{ Intervention group } & \multirow{2}{*}{$\begin{array}{l}\text { Control } \\
\text { groupt }\end{array}$} & \multirow{2}{*}{$\begin{array}{l}\text { Follow- } \\
\text { up } \\
\text { period } \\
\text { (weeks) }\end{array}$} & \multirow{2}{*}{$\begin{array}{l}\text { Body } \\
\text { composition } \\
\text { assessment } \\
\text { method }\end{array}$} & \multirow{2}{*}{$\begin{array}{l}\text { Muscle } \\
\text { strength } \\
\text { assessment } \\
\text { method }\end{array}$} & \multirow{2}{*}{$\begin{array}{l}\text { Physical } \\
\text { performance } \\
\text { assessment } \\
\text { method }\end{array}$} \\
\hline & & Exercise & Nutrition & & & & & \\
\hline $\begin{array}{l}\text { Nagai } \\
\text { (2019), } \\
\text { Japan }\end{array}$ & $\begin{array}{l}\text { I: } 17,72.7 \pm \\
1.4, \text { mixed } \\
(64.7 \%) \\
\text { C: } 19,73.5 \pm \\
2.3, \text { mixed } \\
(68.4 \%)\end{array}$ & $\begin{array}{l}\text { Latex band } \\
\text { training, } \\
\text { squat, and } \\
\text { tai chi, } 90 \\
\text { min } \\
\text { sessions, } \\
\text { once a week }\end{array}$ & $\begin{array}{l}60 \mathrm{mg} \text { maslinic acid, } \\
\text { once a day }\end{array}$ & $\begin{array}{l}\text { Placebo: } \\
\text { jelly without } \\
\text { maslinic } \\
\text { acid }\end{array}$ & 12 & $\begin{array}{l}\text { BMI } \\
\text { Body mass } \\
\text { Fat mass } \\
\text { Fat free } \\
\text { mass } \\
\text { Skeletal } \\
\text { muscle } \\
\text { mass } \\
\text { Segmental } \\
\text { muscle } \\
\text { mass }\end{array}$ & $\begin{array}{l}\text { Grip } \\
\text { strength }\end{array}$ & $\begin{array}{l}\text { Chair stand } \\
\text { Gait speed }\end{array}$ \\
\hline $\begin{array}{l}\text { Nakayama } \\
\text { (2020), } \\
\text { Japan }\end{array}$ & $\begin{array}{l}\text { I: } 61,71.4 \pm \\
6.2, \text { mixed } \\
(74 \%) \\
\text { C: } 61,70.4 \pm \\
5.5, \text { mixed } \\
(77 \%)\end{array}$ & $\begin{array}{l}\text { Body weight } \\
\text { exercises } \\
\text { and } 5 \\
\text { medicine } \\
\text { ball } \\
\text { exercises, } \\
\text { daily }\end{array}$ & $\begin{array}{l}\text { Low-dose milk protein: } \\
10.1 \mathrm{~g} \text { protein, once a } \\
\text { day }\end{array}$ & $\begin{array}{l}\text { Placebo: } \\
\text { Isocaloric } \\
\text { carbohydrate }\end{array}$ & 24 & $\begin{array}{l}\text { Body mass } \\
\text { Fat mass } \\
\text { Lean body } \\
\text { mass }\end{array}$ & $\begin{array}{l}\text { Grip } \\
\text { strength } \\
\text { Knee } \\
\text { extension } \\
\text { Knee } \\
\text { flexion } \\
\text { Push up }\end{array}$ & $\begin{array}{l}\text { Chair stand } \\
\text { Gait speed } \\
\text { TUG }\end{array}$ \\
\hline $\begin{array}{l}\text { Nilsson } \\
\text { (2020), } \\
\text { Canada }\end{array}$ & $\begin{array}{l}\text { I: } 16,77.4 \pm \\
11.2, \text { male } \\
\text { C: } 16,74.4 \pm \\
5.2, \text { male }\end{array}$ & $\begin{array}{l}\text { Home based } \\
\text { resistance } \\
\text { training with } \\
\text { elastic } \\
\text { bands, } 3 \\
\text { times a } \\
\text { week }\end{array}$ & $\begin{array}{l}\text { Multi nutrients: } 24 \mathrm{~g} \\
\text { whey protein, } 16 \mathrm{~g} \\
\text { micellar casein } \\
\text { contained } 416 \mathrm{mg} \\
\text { calcium, } 3 \mathrm{~g} \text { creatine, } \\
\text { vitamin D } 1000 \mathrm{IU} \text {, and } \\
\text { omega- } 3 \text { fish-oil } \\
\text { containing } 1.51 \mathrm{~g} \text { EPA } \\
\text { and } 0.95 \mathrm{~g} \text { DHA, once a } \\
\text { day }\end{array}$ & $\begin{array}{l}\text { Placebo: } \\
\text { collagen and } \\
\text { sunflower oil }\end{array}$ & 12 & $\begin{array}{l}\text { ASMM } \\
\text { Body mass } \\
\text { BMI } \\
\text { Lean body } \\
\text { mass }\end{array}$ & $\begin{array}{l}\text { Grip } \\
\text { strength } \\
\text { Knee } \\
\text { extension } \\
\text { Leg press }\end{array}$ & $\begin{array}{l}\text { Chair stand } \\
\text { SPPB } \\
\text { Stair climb } \\
\text { TUG } \\
4 \mathrm{~m} \text { walk }\end{array}$ \\
\hline $\begin{array}{l}\text { Seino } \\
\text { (2018), } \\
\text { Japan }\end{array}$ & $\begin{array}{l}\text { I: } 40,73.4 \pm \\
4.3, \text { mixed } \\
(85 \%) \\
\text { C: } 40,73.7 \pm \\
4.3, \text { mixed } \\
(82.5 \%)\end{array}$ & $\begin{array}{l}\text { Weight- } \\
\text { bearing } \\
\text { exercise and } \\
\text { exercises } \\
\text { using a } \\
\text { resistance } \\
\text { band and } \\
\text { Pilates ball, } \\
\text { 60min } \\
\text { sessions, } \\
\text { twice a } \\
\text { week }\end{array}$ & $\begin{array}{l}\text { Fortified milk } \\
\text { containing } 10.5 \mathrm{~g} \text { total } \\
\text { milk protein, } 3.9 \mathrm{~g} \text { fat, } \\
9.3 \mathrm{~g} \text { carbohydrate, and } \\
337 \text { mg calcium at } \\
\text { lunch and micronutrient } \\
\text { beverage at breakfast, } \\
\text { daily }\end{array}$ & Usual diet & 12 & $\begin{array}{l}\text { Body mass } \\
\text { Lean body } \\
\text { mass } \\
\text { Lower limb } \\
\text { lean mass } \\
\text { SMI }\end{array}$ & $\begin{array}{l}\text { Grip } \\
\text { strength } \\
\text { Knee } \\
\text { extension }\end{array}$ & $\begin{array}{l}\text { Chair stand } \\
\text { Gait speed } \\
\text { One leg } \\
\text { standing } \\
\text { with eyes } \\
\text { open } \\
\text { TUG }\end{array}$ \\
\hline $\begin{array}{l}\text { Stout } \\
\text { (2013), USA }\end{array}$ & $\begin{array}{l}\text { l: } 24,73 \pm 4.9 \\
\text { mixed }(54.2 \%) \\
\text { C: } 24,73 \pm 4.9 \\
\text { mixed }(54.2 \%)\end{array}$ & $\begin{array}{l}\text { Resistance } \\
\text { exercise, } \\
\text { three times } \\
\text { a week for } \\
21 \text { weeks }\end{array}$ & $\begin{array}{l}1.5 \mathrm{~g} \text { calcium and } 4 \mathrm{~g} \\
\text { carbohydrate, twice a } \\
\text { day }\end{array}$ & $\begin{array}{l}\text { Placebo: } \\
\text { 200mg } \\
\text { calcium and } \\
4 \mathrm{~g} \\
\text { carbohydrate }\end{array}$ & 24 & $\begin{array}{l}\text { Lean body } \\
\text { mass } \\
\text { Lower limb } \\
\text { lean mass } \\
\text { Upper limb } \\
\text { lean mass }\end{array}$ & $\begin{array}{l}\text { Grip } \\
\text { strength }\end{array}$ & Chair stand \\
\hline $\begin{array}{l}\text { Sugihara } \\
\text { (2018), } \\
\text { Brazil }\end{array}$ & $\begin{array}{l}\text { I: } 15,67.4 \pm \\
\text { 4.1, female } \\
\text { C: } 16,67.8 \pm \\
4.1, \text { female }\end{array}$ & $\begin{array}{l}\text { Resistance } \\
\text { training } \\
\text { using a } \\
\text { combination } \\
\text { of free } \\
\text { weights and } \\
\text { machines, } \\
45 \sim 50 \mathrm{~min} \\
\text { sessions, } 3 \\
\text { times a } \\
\text { week }\end{array}$ & $\begin{array}{l}35 \mathrm{~g} \text { whey protein, } \\
\text { immediately after each } \\
\text { resistance training }\end{array}$ & $\begin{array}{l}\text { Placebo: } \\
35 \mathrm{~g} \\
\text { maltodextrin }\end{array}$ & 12 & $\begin{array}{l}\text { Body mass } \\
\text { BMI } \\
\text { Lower limb } \\
\text { lean mass } \\
\text { SMI } \\
\text { Upper limb } \\
\text { lean mass }\end{array}$ & $\begin{array}{l}\text { Biceps curl } \\
\text { Chest press } \\
\text { Knee } \\
\text { extension }\end{array}$ & - \\
\hline
\end{tabular}

†The resistance exercise of the control group applied in the same manner as in the experimental group.

† The duration of intervention in most studies were the same as the follow-up period.

‡ ASMM: appendicular skeletal muscle mass, BMI : body mass index, C: control group, DHA: docosahexaenoic acid, EPA: eicosapentaenoic acid, I: intervention group, SMl; skeletal muscle massi index, SPPB: short physical performance battery, TUG: time up and go 


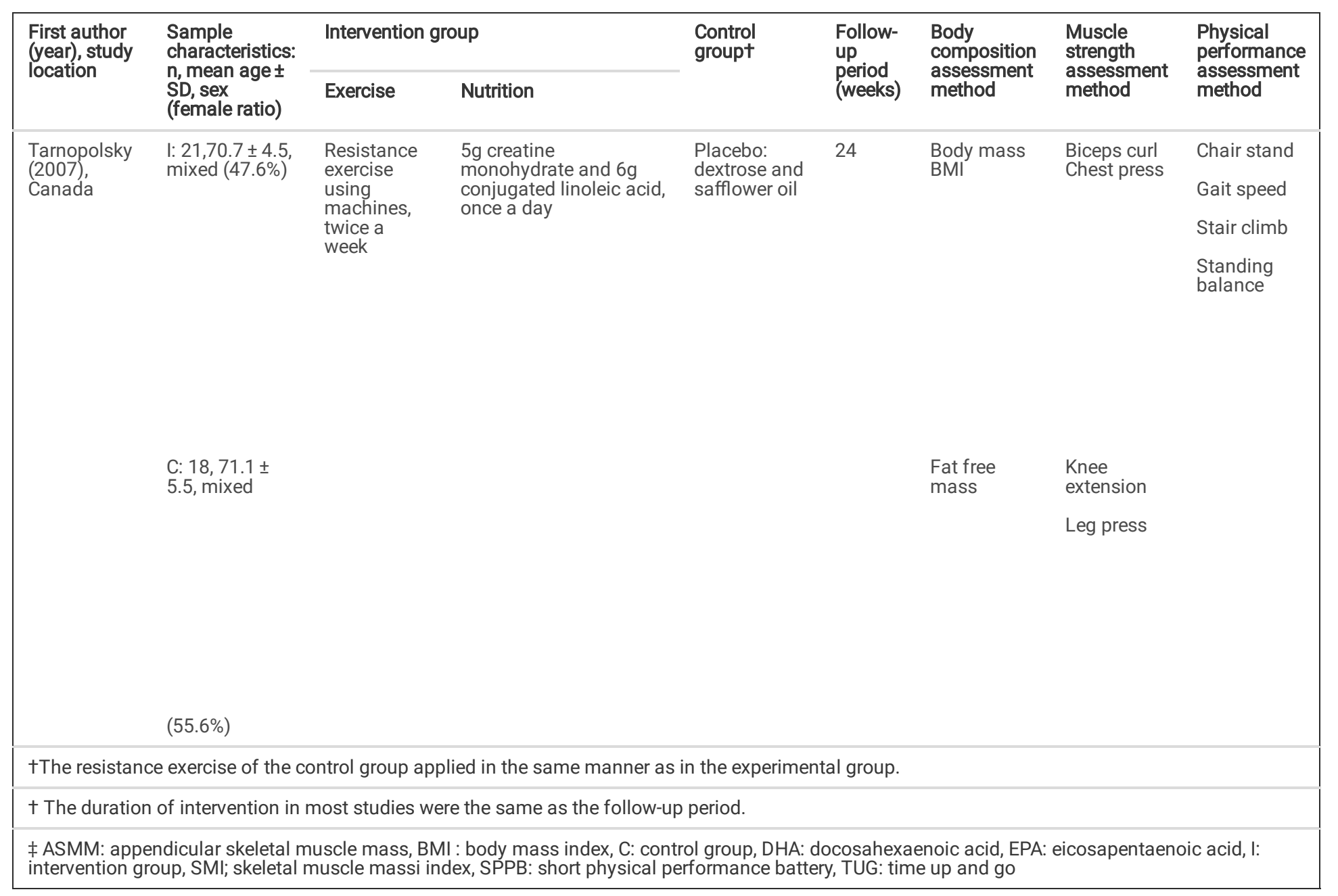

All of the studies administered supervised exercise programs except one study [27], which included a home-based exercise program with consistent encouragement. The exercise programs included resistance training in 21 studies and multimodal exercise including aerobic, balance, or functional training and resistance training in 5 studies. In almost all the studies, the exercise programs were performed twice ( 7 studies) or three times ( 15 studies) a week on nonconsecutive days; the exercise programs were performed daily in two studies, once a week in one study, and biweekly in one study.

The RCTs provided protein (ten studies), creatine (three studies), long chain n-3 polyunsaturated fatty acids (PUFA omega-3) (two studies), calcium (a study), maslinic acid (a study), vitamin D (two studies), vitamins C and E (two studies), linoleic acid and creatine (a study), and multinutrients containing more than three nutrients (four studies). Most studies provided nutritional supplements in pill, capsule, powder or drink forms. A study provided a diet with red meat [28], and another study provided a personalized and nutritionally balanced diet [29]. Most studies provided the control groups with an isocaloric placebo. Three studies provided the control groups with pills or capsules containing some nutrients, such as calcium or omega-3 [30-32]. The intervention period ranged between 12 and 36 weeks: 12 weeks in 12 studies, 14 weeks in one study, 16 weeks in one study, 18 weeks in one study, 24 weeks in ten studies, and 36 weeks in one study.

\section{Risk of Bias}

The risk of bias results for the 26 RCTs are demonstrated in Fig. 2. Regarding the randomization process, eight studies had a low risk of bias, 17 had some concerns, and one study had a high risk of bias because of a failure to conceal group allocation. Regarding deviation from the intended intervention, three had some concerns, and the others had a low risk of bias. As there were no studies in which missing values were judged to have an impact on the study results, all studies had a low risk of bias in the domain of missing outcome data. All studies had a low risk of bias in the domain of measurement of outcome, either because the outcome assessor was blinded or the outcome assessor's awareness of the group assignments was judged to not affect the measurement of muscle mass, strength, or physical function. In the fifth domain, the selection of the reported results, 11 studies had a low risk of bias, while the other 15 studies had some concerns because of the absence of a prespecified trial protocol. Overall, six RCTs had a low risk of bias, 19 RCTs had some concerns, and one study had a high risk of bias.

Effects of Resistance Training and Nutritional Interventions Compared with Those of Resistance Training Only on Muscle Mass, Muscle Strength, and Physical Functional Performance

The effect sizes and 95\% confidence intervals (Cls) for individual studies and all studies are shown in Fig. 3 . The results of the meta-analysis showed no significant effects on lean body mass ( $n=13, \mathrm{MD} 0.12, \mathrm{Cl}-0.46$ to 0.7$)$, appendicular skeletal muscle mass $(n=6, \mathrm{MD}-0.01, \mathrm{Cl}-0.26$ to 0.24$)$, hand grip strength ( $n=11, \operatorname{SMD} 0.08, \mathrm{Cl}-0.09$ to 0.24$)$, knee extension strength $(n=16, \operatorname{SMD} 0.08, \mathrm{Cl}-0.05$ to 0.21$)$, the chair stand test results $(n=7, \mathrm{MD}-0.13, \mathrm{Cl}$ 
-0.44 to 0.17 ), or the timed up-and-go test results ( $n=9, \mathrm{MD}$ zero, $\mathrm{Cl}-0.17$ to 0.17 ). The $\mathrm{I}^{2}$ values for all outcomes except lean body mass were zero, indicating that heterogeneity was low for these outcomes and lean body mass had moderate heterogeneity $\left(\mathrm{I}^{2}=40 \%\right)$.

\section{Subgroup Analysis According to the Characteristics of the Participants and Interventions}

The results of the subgroup analyses are shown in Tables 2-4. The subgroup analyses for lean body mass showed significant differences between the types of nutritional interventions $\left(\mathrm{Chi}^{2}=9.02, p=.01\right)$. Among the nutritional interventions, only those with creatine showed significant effects on lean body mass ( $n=3, M D 2.96, \mathrm{Cl} 0.76$ to 5.16 ). Regarding the other subgroup analyses, there were no significant differences according to the mean age and sex of the participants, type of nutritional intervention, or duration of intervention.

Table 2

Summary of over effects and subgroup analyses results for muscle mass

\begin{tabular}{|c|c|c|c|c|c|c|c|c|c|c|}
\hline \multirow[t]{2}{*}{ Subgroups } & \multicolumn{5}{|c|}{ Lean body mass } & \multicolumn{5}{|c|}{ Appendicular skeletal muscle mass } \\
\hline & $\mathbf{n}$ & MD & $95 \% \mathrm{Cl}$ & $1^{2}$ & Subgroup differences & $\mathbf{n}$ & MD & $95 \% \mathrm{Cl}$ & $1^{2}$ & Subgroup differences \\
\hline Overall & 13 & 0.12 & $-0.46 \sim 0.7$ & $40 \%$ & - & 6 & -0.01 & $-0.26 \sim 0.24$ & $0 \%$ & - \\
\hline \multicolumn{11}{|c|}{ Participant mean age } \\
\hline$<70$ & 5 & 0.39 & $-1.58 \sim 2.35$ & $57 \%$ & \multirow[t]{2}{*}{$\chi^{2}=0.07(p=.79)$} & 2 & -0.89 & $-2.46 \sim 0.68$ & $0 \%$ & \multirow[t]{2}{*}{$\chi^{2}=1.24(p=.27)$} \\
\hline$\geq 70$ & 8 & 0.11 & $-0.40 \sim 0.62$ & $32 \%$ & & 4 & 0.01 & $-0.24 \sim 0.27$ & $0 \%$ & \\
\hline \multicolumn{11}{|l|}{ Participant sex } \\
\hline Male & 8 & -0.42 & $-2.73 \sim 1.88$ & $25 \%$ & \multirow[t]{3}{*}{$\chi^{2}=0.86(p=.65)$} & 2 & -0.04 & $-2.27 \sim 2.19$ & $65 \%$ & \multirow[t]{3}{*}{$\chi^{2}=0.00(p=.97)$} \\
\hline Female & 3 & 0.89 & $-1.10 \sim 2.88$ & $58 \%$ & & 0 & - & - & - & \\
\hline Mixed & 4 & 0.03 & $-0.24 \sim 0.31$ & $0 \%$ & & 4 & -0.00 & $-0.26 \sim 0.26$ & $0 \%$ & \\
\hline \multicolumn{11}{|l|}{ Nutrition type } \\
\hline Creatine & 3 & 2.96 & $0.76 \sim 5.16$ & $10 \%$ & \multirow[t]{3}{*}{$\chi^{2}=9.02(p=.01)$} & - & - & - & - & \multirow[t]{3}{*}{$\chi^{2}=0.62(p=.43)$} \\
\hline Multi-nutrients & 3 & 0.19 & $-0.16 \sim 0.55$ & $0 \%$ & & 2 & 0.39 & $-0.68 \sim 1.45$ & $0 \%$ & \\
\hline Protein & 5 & -0.18 & $-0.54 \sim 0.17$ & $0 \%$ & & 2 & -0.20 & $-1.23 \sim 0.82$ & $47 \%$ & \\
\hline \multicolumn{11}{|c|}{ Duration of intervention } \\
\hline$\leq 14$ & 10 & 0.47 & $-0.64 \sim 1.55$ & $51 \%$ & \multirow[t]{2}{*}{$\chi^{2}=0.24(p=.62)$} & 4 & 0.08 & $-0.23 \sim 0.40$ & $0 \%$ & \multirow[t]{2}{*}{$\chi^{2}=1.02(p=.31)$} \\
\hline$\geq 16$ & 3 & 0.18 & $-0.18 \sim 0.53$ & $0 \%$ & & 2 & -0.19 & $-0.63 \sim 0.24$ & $0 \%$ & \\
\hline
\end{tabular}


Table 3

Summary of over effects and subgroup analyses results for muscle strength

\begin{tabular}{|c|c|c|c|c|c|c|c|c|c|c|}
\hline \multirow[t]{2}{*}{ Subgroups } & \multicolumn{5}{|c|}{ Hand grip strength } & \multicolumn{5}{|c|}{ Knee extension strength } \\
\hline & $\mathbf{n}$ & SMD & $95 \% \mathrm{Cl}$ & $\left.\right|^{2}$ & Subgroup differences & $\mathbf{n}$ & SMD & $95 \% \mathrm{Cl}$ & $p^{2}$ & Subgroup differences \\
\hline Overall & 11 & 0.08 & $-0.09 \sim 0.24$ & $0 \%$ & - & 16 & 0.08 & $-0.05 \sim 0.21$ & $0 \%$ & - \\
\hline \multicolumn{11}{|c|}{ Participant mean age } \\
\hline$<70$ & 4 & 0.10 & $-0.21 \sim 0.41$ & $0 \%$ & \multirow[t]{2}{*}{$\chi^{2}=0.02(p=.88)$} & 7 & 0.07 & $-0.21 \sim 0.35$ & $26 \%$ & \multirow[t]{2}{*}{$\chi^{2}=0.00(p=.96)$} \\
\hline$\geq 70$ & 7 & 0.07 & $-0.13 \sim 0.27$ & $0 \%$ & & 9 & 0.09 & $-0.06 \sim 0.25$ & $0 \%$ & \\
\hline \multicolumn{11}{|l|}{ Participant sex } \\
\hline Male & 5 & 0.08 & $-0.36 \sim 0.53$ & $38 \%$ & \multirow[t]{3}{*}{$\chi^{2}=0.31(p=.86)$} & 6 & 0.04 & $-0.27 \sim 0.35$ & $0 \%$ & \multirow[t]{3}{*}{$\chi^{2}=0.80(p=.67)$} \\
\hline Female & 4 & -0.06 & $-0.44 \sim 0.31$ & $0 \%$ & & 6 & 0.20 & $-0.14 \sim 0.53$ & $18 \%$ & \\
\hline Mixed & 5 & 0.04 & $-0.18 \sim 0.27$ & $0 \%$ & & 7 & 0.06 & $-0.11 \sim 0.22$ & $0 \%$ & \\
\hline \multicolumn{11}{|l|}{ Nutrition type } \\
\hline Multi-nutrients & 2 & -0.04 & $-0.48 \sim 0.40$ & $21 \%$ & \multirow[t]{3}{*}{$\chi^{2}=0.10(p=.75)$} & 2 & 0.05 & $-0.36 \sim 0.46$ & $12 \%$ & \multirow[t]{3}{*}{$\chi^{2}=1.27(p=53)$} \\
\hline Protein & 5 & 0.04 & $-0.19 \sim 0.26$ & $0 \%$ & & 7 & -0.02 & $-0.20 \sim 0.16$ & $0 \%$ & \\
\hline Vitamin D & - & - & - & - & & 2 & 0.20 & $-0.14 \sim 0.54$ & $0 \%$ & \\
\hline \multicolumn{11}{|c|}{ Duration of intervention } \\
\hline$\leq 14$ & 5 & 0.14 & $-0.13 \sim 0.41$ & $0 \%$ & \multirow[t]{2}{*}{$\chi^{2}=0.34(p=.56)$} & 8 & 0.11 & $-0.08 \sim 0.31$ & $4 \%$ & $\chi^{2}=0.17(p=.68)$ \\
\hline$\geq 16$ & 6 & 0.04 & $-0.17 \sim 0.25$ & $0 \%$ & & 8 & 0.06 & $-0.13 \sim 0.24$ & $0 \%$ & \\
\hline
\end{tabular}

Table 4

Summary of over effects and subgroup analyses results for physical functional performance

\begin{tabular}{|c|c|c|c|c|c|c|c|c|c|c|}
\hline \multirow[t]{2}{*}{ Subgroups } & \multicolumn{5}{|c|}{ Chair stand test } & \multicolumn{5}{|c|}{ Timed up and go test } \\
\hline & $\mathbf{n}$ & MD & $95 \% \mathrm{Cl}$ & $p^{2}$ & Subgroup differences & $\mathbf{n}$ & MD & $95 \% \mathrm{Cl}$ & $\left.\right|^{2}$ & Subgroup differences \\
\hline Overall & 7 & -0.13 & $-0.44 \sim 0.17$ & $0 \%$ & - & 9 & 0.00 & $-0.17 \sim 0.17$ & $0 \%$ & - \\
\hline \multicolumn{11}{|c|}{ Participant mean age } \\
\hline$<70$ & 2 & -0.44 & $-1.08 \sim 0.20$ & $0 \%$ & \multirow[t]{2}{*}{$\chi^{2}=1.11(p=.29)$} & 1 & 0.01 & $-0.17 \sim 0.18$ & $0 \%$ & \multirow[t]{2}{*}{$\chi^{2}=0.06(p=.80)$} \\
\hline$\geq 70$ & 5 & -0.05 & $-0.39 \sim 0.30$ & $0 \%$ & & 8 & 0.00 & $-0.17 \sim 0.17$ & $0 \%$ & \\
\hline \multicolumn{11}{|l|}{ Participant sex } \\
\hline Male & 3 & -0.35 & $-0.95 \sim 0.25$ & $0 \%$ & \multirow[t]{3}{*}{$\chi^{2}=1.17(p=.56)$} & 3 & 0.23 & $-0.25 \sim 0.72$ & $0 \%$ & \multirow[t]{3}{*}{$\chi^{2}=1.00(p=.32)$} \\
\hline Female & 2 & -0.39 & $-1.27 \sim 0.49$ & $0 \%$ & & 0 & - & - & - & \\
\hline Mixed & 3 & -0.02 & $-0.40 \sim 0.35$ & $0 \%$ & & 6 & -0.03 & $-0.22 \sim 0.15$ & $0 \%$ & \\
\hline \multicolumn{11}{|l|}{ Nutrition type } \\
\hline Multi-nutrients & 2 & 0.15 & $-0.41 \sim 0.72$ & $0 \%$ & \multirow[t]{2}{*}{$\chi^{2}=1.1(p=.29)$} & 3 & 0.13 & $-0.25 \sim 0.52$ & $9 \%$ & \multirow[t]{2}{*}{$\chi^{2}=0.73(p=.39)$} \\
\hline Protein & 3 & -0.22 & $-0.63 \sim 0.19$ & $0 \%$ & & 3 & -0.06 & $-0.28 \sim 0.16$ & $0 \%$ & \\
\hline \multicolumn{11}{|c|}{ Duration of intervention } \\
\hline$\leq 14$ & 4 & -0.10 & $-0.53 \sim 0.32$ & $0 \%$ & \multirow[t]{2}{*}{$\chi^{2}=0.04(p=.84)$} & 5 & 0.07 & $-0.17 \sim 0.31$ & $0 \%$ & \multirow[t]{2}{*}{$\chi^{2}=0.62(p=.43)$} \\
\hline$\geq 16$ & 3 & -0.16 & $-0.60 \sim 0.27$ & $0 \%$ & & 4 & -0.07 & $-0.31 \sim 0.17$ & $0 \%$ & \\
\hline
\end{tabular}

\section{Sensitivity Analysis}

Sensitivity analysis was conducted to compare the effect sizes, Cls, and $\mathrm{I}^{2}$ values by excluding three studies that provided some nutrients to control groups. There were no significant differences in lean body mass ( $n=12, \mathrm{MD} 0.15, \mathrm{Cl}-0.46$ to 0.75$)$, appendicular skeletal muscle mass ( $\mathrm{n}=5, \mathrm{MD} 0.09, \mathrm{Cl}$ -0.23 to 0.40 ), hand grip strength ( $n=9, M D 0.07, C l-0.11$ to 0.25$)$, knee extension ( $n=14, M D 0.09, C l-0.04$ to 0.23 ), chair stand test results ( $n=11, M D$ $-0.05, \mathrm{Cl}-0.2$ to 0.1 ), or timed up-and-go test results ( $\mathrm{n}=6, \mathrm{MD}$ zero, $\mathrm{Cl}-0.18$ to 0.18 ). The $\mathrm{I}^{2}$ values for all outcomes except for lean body mass were zero, indicating that heterogeneity was low for these outcomes and lean body mass had moderate heterogeneity $\left(I^{2}=44 \%\right)$. 


\section{Discussion}

Nutrient-dense foods that ensure sufficient intake of energy, protein and micronutrients are important to prevent frailty and sarcopenia and promote physical activity. However, to date, the optimal type of nutritional intervention or supplementation is unclear for the prevention of frailty and sarcopenia. This study was conducted to compare the synergistic effect of nutritional interventions combined with resistance training with that of resistance training only. This study was conducted to provide insight into resource optimization and strategies to prevent frailty and sarcopenia.

This systematic review and meta-analysis showed that there were no additional effects of nutritional interventions when combined with resistance training on muscle mass, strength, or physical function. Of note, in three studies, the control conditions included some nutrients that have biological benefits [3032], which likely reduced the calculated effect size when the data for the control conditions were pooled. However, the findings of the sensitivity analysis showed little possibility of blunted effects. One of the possible reasons for this lack of significant results is that the analysis included studies of healthy older adults who might not have nutrient deficiencies with the usual diets [23]. Healthy diets provide a broad range of micronutrients and bioactive nonnutrients as well as macronutrients that might not be included in the experimental supplements in trials. In addition, since diets are patterned, isolating the effects of individual experimental supplements might not be possible without controlling the usual diet. Thus, the effects of nutritional interventions might be blunted among older adults who habitually consume sufficient nutrients. However, in previous studies that provided vitamin D-deficient and mobility-limited older adults with a protein mixture containing $20 \mathrm{~g}$ protein, $800 \mathrm{lU}$ vitamin D, $350 \mathrm{mg}$ calcium, and other minerals once a day for six months with an exercise program, there were no differences in muscle function parameters such as leg strength, gait speed, and short physical performance battery between this group and the exercise-only control group except in muscle density [33, 34]. In another study that also provided sarcopenic older adults who had low protein intake with multinutrient supplements containing $21 \mathrm{~g}$ protein, $800 \mathrm{IU}$ vitamin D and other nutrients once a day for three months with an exercise program, there were no differences between the two groups, although both groups exhibited improved muscle function [33]. It is necessary to additionally consider the dose of the nutrient and duration of intervention and monitor dietary energy intake. Despite the lack of evidence, greater benefits of resistance training along with nutritional supplementation are expected in older adults who already have muscle failure or habitually have low nutrient intake.

In the subgroup analysis of the types of nutrients, only creatine showed significant effects on lean body mass. All three studies included in this metaanalysis administered $5 \mathrm{~g}$ creatine daily combined with resistance training 3 times a week for 12 weeks [35, 36] or twice a week for24 weeks [37]. Recent systematic reviews similarly identified the additive effect of creatine during resistance training on body composition, muscle strength, and physical function [38, 39]. As skeletal muscle has no capacity for creatine biosynthesis, the consumption of creatine-containing food or supplementation of creatine increases creatine and phosphocreatine levels in skeletal muscle and elevates phosphate resynthesis (energy buffer) during high-energy demanded exercise, such as repetitive resistance training [10,40,41]. Creatine helps to increase muscle mass and strength by indirectly increasing work capacity, and the combination of creatine supplementation and resistance training promotes muscle protein synthesis. Alternatively, creatine supplementation may enhance muscle protein synthesis stimulating signaling pathways (myogenic regulatory factors), which facilitate myosatellite cell proliferation and differentiation [42]. Controversy exists as to whether creatine stores and metabolism are affected by aging, but creatine supplements can account for dietary changes and reductions in physical activity with aging [39]. The effects sizes for variables other than lean body mass were not significant in this study. Additional meta-analyses including more experimental studies are needed to verify the effects of creatine on muscle mass and function in older adults.

As proteins provide amino acids that are essential for the muscle protein synthesis and act as anabolic stimuli, protein consumption increases muscle mass, and protein consumption following resistance training enhances net protein utilization, attenuating exercise-induced muscle protein breakdown [41, 43]. The combination of a nutritional intervention and exercise was expected to have a synergistic effect on muscle function, but the findings of this study did not support this hypothesis. On the other hand, in a previous meta-analysis, protein supplements for sarcopenic older adults along with exercise showed a larger effect size than did exercise alone and no intervention [25]. The previous meta-analysis was conducted in frail, sarcopenic, or mobilitylimited older adults and included not only community-dwelling older adults but also institutionalized older adults. Individuals with existing nutritional deficiencies or muscle failure might have been shown to respond better to accompanying nutritional supplements than to exercise alone. Additional studies are needed to determine whether the inconsistency in findings resulted from the characteristics of the subjects.

Muscle protein synthesis through protein intake in older adults should be maximized with consideration of the frequency, distribution, and other nutritional components, such as creatine, vitamins, and fatty acids [22,41]. It is recommended that older adults consume $\geq 0.4 \mathrm{~g} / \mathrm{kg}$ per meal and $1.2-1.6 \mathrm{~g} / \mathrm{kg}$ per day to induce muscle protein synthesis saturation to thus support muscle function [41]. Among the included studies, two studies provided an appropriate amount of protein ( $10 \mathrm{~g}$ milk protein and $0.5 \mathrm{~g} / \mathrm{kg}$ whey protein) three times a day, taking into account frequency and distribution [21, 25, 26]. Other studies provided $10.1 \mathrm{~g}-25 \mathrm{~g}$ protein once a day [44-47] or $20 \mathrm{~g} \sim 35 \mathrm{~g}$ protein 3 times a week on the days exercise was performed [48-50]. A previous review showed that multi-ingredient protein supplements have the potential to increase the benefits of resistance training, but there were no differences in the effects on muscle mass and strength between multi-ingredient protein and single protein [51]. The impact of multiple nutrients is unclear, as there are complex interactions between food components inducing potential synergistic effects, so nutritional interventions involving dietary modifications with various and balanced nutrients or whole food approaches rather than a single specific nutrient can be effective in improving muscle mass and function [52]. Among the 26 RCTs, five provided multinutrients that were arbitrarily defined as containing three or more nutrients. Of the five studies, only two used a whole-food or whole-diet approach. The number of studies was too small to verify the effect of the whole-food or whole-diet approach.

Nutritional effects may not manifest following dietary interventions of short durations. Although this study showed that there were no differences in effect sizes according to the intervention period, a 6-year longitudinal study showed a positive relationship between daily protein intake and muscle strength [53]; nutritional contributions can be expected to be observed in the long term. Thus, despite the nonsignificant results, nutritional interventions may still be

Page 12/17 
beneficial for older adults who do not lack nutrients. With aging, muscle loss (breakdown) occurs more rapidly than does muscle synthesis, so additional supplements may be required. In addition, older adults experience declines in food intake because of changes in appetite and a lack of hunger, which is referred to as 'anorexia of aging' [54]. As consumed food is metabolized to synthesize energy for organ function, poor nourishment leads to body fat and muscle being catabolized to provide energy. Not only a lack of specific nutrients but also the consumption of an insufficient amount of food contributes to weight loss and declines in muscle mass, strength and physical function, which can lead to physical frailty and sarcopenia. Thus, the consumption of an adequate amount of food containing nutrients essential for muscle function is important to maintain muscle mass, strength, and physical function [22, 55]. Considering changes occur in various physiological functions as well as muscle function, interventions with a balanced diet are important in older people. As nutritional interventions have the advantages of low costs and high availability and accessibility, additional studies are necessary to determine whether they can be effective in preventing frailty and sarcopenia.

This study has several limitations. First, this meta-analysis included only retrievable RCTs that were published in English, which may have contributed to language bias. Second, this study in healthy older adults might not have demonstrated significant effects on muscle mass, muscle strength, and physical function due to the ceiling effect. Additional systematic reviews and meta-analyses are needed to identify the additional effects of nutritional interventions when combined with resistance training among dynapenic, sarcopenic, or frail older adults. Third, as mentioned above, the amount, frequency, and distribution of nutrients administered are important to consider to fully assess the effects of nutritional interventions; however, these factors were not assessed in the meta-analysis.

As the levels of variability in muscle mass and functional measurements are quite high in older adults, it is hard to obtain adequate statistical power to verify differences between groups in many studies on nutritional interventions. This meta-analysis showed that nutritional interventions have no additional effect on body composition, muscle strength, or physical function when combined with resistance training. Only creatine showed synergistic effects with resistance training on muscle mass. The enhanced effect of nutritional interventions for unhealthy older adults, such as frail, sarcopenic, nutritionally deficient older adults, needs to be investigated in future studies. The long-term effects of nutrition on muscle function also need to be studied. In addition, additional studies should be conducted to identify the dietary parameters that maximize nutritional effects on muscle protein synthesis, including dose, frequency, distribution, and recipes that take into account interactions with other nutrients. Health-promoting interventions such as exercise and diet are important for at-risk older adults to prevent clinically evident disability. This systematic review and meta-analysis provides a comprehensive synthesis of the experimental results available to date for health practitioners and researchers to establish intervention strategies or public health policies.

\section{Abbreviations}

Cl: confidence interval; IU: international unit; MD: Mean difference; PUFA: Polyunsaturated fatty acid; RCT: Randomized controlled trial; RoB: Risk of bias; SD: Standard deviation; SMD Standardized mean difference; UK: United Kingdom; USA: United States of America

\section{Declarations}

\section{Ethics Approval and Consent to Participate}

This study was approved for a review exemption from the institutional review board of a university, Chuncheon, Korea (KWNUIRB-2020-07-006).

\section{Consent for Publication}

Not applicable.

\section{Availability of Data and Materials}

The authors can confirm that all relevant data are included in the article.

\section{Funding}

This work was supported by the National Research Foundation of Korea (NRF) grant funded by the Korean government (MSIT) (No. 2020R1G1A1003901).

\section{Authors' Contributions}

MK designed this study, collected and selected articles, extracted data from included studies, evaluated the risk of bias, performed meta-analyses, and draft the manuscript. HY performed data collection, selection of studies according to criteria, and data extraction. JY evaluated the risk of bias of included studies, checked the results of meta-analyses, helped to draft the manuscript. All authors read and approved the final manuscript.

\section{Author's information}

${ }^{1}$ MoonKi Choi is an Assistant professor in College of Nursing, Kangwon National University, Chuncheon-si, Gangwon-do, Republic of Korea, 24341

${ }^{2}$ Hayeon Kim is an undergraduate nursing students at Seoul Women's College of Nursing, Ganhodae-ro 38, Seodaemun-gu, Seoul, Republic of Korea, 03617

${ }^{3}$ Juyeon Bae is an Assiatant Professor in Department of Nursing, Yeoju Institute of Technology, Sejong-ro 338, Yeoju-si, Gyeonggi-do, Korea 


\section{Acknowledgements}

Not applicable.

\section{References}

1. World Health Organization. Active ageing: a policy framework. Geneva, Switzerland: World Health Organization; 2002.

2. Clegg A, Young J, Iliffe S, Rikkert MO, Rockwood K. Frailty in elderly people. Lancet. 2013;381:752-62.

3. Fried LP, Tangen CM, Walston J, Newman AB, Hirsch C, Gottdiener J, et al. Frailty in older adults: evidence for a phenotype. J Gerontol A Biol Sci Med Sci. 2001; $56: 146-56$.

4. Rockwood K, Mitnitski A. Frailty in relation to the accumulation of deficits. J Gerontol A Biol Sci Med Sci. 2007;62:722-7.

5. Rodríguez-Mañas L, Féart C, Mann G, Viña J, Chatterji S, Chodzko-Zajko W, et al. Searching for an operational definition of frailty: a Delphi method based consensus statement: the frailty operative definition-consensus conference project. J Gerontol A Biol Sci Med Sci. $2013 ; 68: 62$ - 7.

6. Hoogendijk EO, Afilalo J, Ensrud KE, Kowal P, Onder G, Fried LP. Frailty: implications for clinical practice and public health. Lancet. 2019;394:1365-75.

7. Shmuel S, Lund JL, Alvarez C, Hsu CD, Palta P, Kucharska-Newton A, et al. Polypharmacy and incident frailty in a longitudinal community-based cohort study. J Am Geriatr Soc. 2019;67:2482-9.

8. Yadav UN, Tamang MK, Thapa TB, Hosseinzadeh H, Harris MF, Yadav KK. Prevalence and determinants of frailty in the absence of disability among older population: a cross sectional study from rural communities in Nepal. BMC Geriatr. 2019;19:283.

9. Classification of Disease. 2021 ICD-10-CM diagnosis code M62.84. http://www.icd10data.com/ICD10CM/Codes/M00-M99/M60-M63/M62-/M62.84 (2021). Accessed 15 Feb 2021.

10. Cruz-Jentoft AJ, Bahat G, Bauer J, Boirie Y, Bruyère O, Cederholm T, et al. Sarcopenia: revised European consensus on definition and diagnosis. Age Ageing. 2019;48:16-31.

11. Mayhew AJ, Amog K, Phillips S, Parise G, McNicholas PD, De Souza RJ, et al. The prevalence of sarcopenia in community-dwelling older adults, an exploration of differences between studies and within definitions: a systematic review and meta-analyses. Age Ageing. 2019;48:48-56.

12. Bernabei R, Martone AM, Vetrano DL, Calvani R, Landi F, Marzetti E. Frailty, physical frailty, sarcopenia: a new conceptual model. Stud Health Technol Inform. 2014;203:78-84.

13. Dos Santos L, Cyrino ES, Antunes M, Santos DA, Sardinha LB. Sarcopenia and physical independence in older adults: the independent and synergic role of muscle mass and muscle function. J Cachexia Sarcopenia Muscle. 2017;8:245-50.

14. Schaap LA, Van Schoor NM, Lips P, Visser M. Associations of sarcopenia definitions, and their components, with the incidence of recurrent falling and fractures: the longitudinal aging study Amsterdam. J Gerontol A Biol Sci Med Sci. 2018;73:1199-204.

15. Steffl M, Sima J, Shiells K, Holmerova I. The increase in health care costs associated with muscle weakness in older people without long-term illnesses in the Czech Republic: results from the Survey of Health, Ageing and Retirement in Europe (SHARE). Clin Interv Aging. 2017;12:2003-7.

16. Fairhall N, Sherrington C, Kurrle SE, Lord SR, Lockwood K, Howard K, et al. Economic evaluation of a multifactorial, interdisciplinary intervention versus usual care to reduce frailty in frail older people. J Am Med Dir Assoc. 2015;16:41-8.

17. Frost R, Belk C, Jovicic A, Ricciardi F, Kharicha K, Gardner B, et al. Health promotion interventions for community-dwelling older people with mild or prefrailty: a systematic review and meta-analysis. BMC Geriatr. 2017;17:157.

18. Beaudart C, Dawson A, Shaw SC, Harvey NC, Kanis JA, Binkley N, et al. Nutrition and physical activity in the prevention and treatment of sarcopenia: systematic review. Osteoporos Int. 2017;28:1817-33.

19. Daly RM. Exercise and nutritional approaches to prevent frail bones, falls and fractures: an update. Climacteric. 2017;20:119-24.

20. Hita-Contreras F, Bueno-Notivol J, Martínez-Amat A, Cruz-Díaz D, Hernandez AV, Pérez-López FR. Effect of exercise alone or combined with dietary supplements on anthropometric and physical performance measures in community-dwelling elderly people with sarcopenic obesity: a meta-analysis of randomized controlled trials. Maturitas. 2018;116:24-35.

21. Dulac MC, Pion CH, Lemieux FC, Pinheiro Carvalho L, El Hajj Boutros G, Bélanger M, et al. Effects of slow- v. fast-digested protein supplementation combined with mixed power training on muscle function and functional capacities in older men. Br J Nutr. 2020;125:1017-33.

22. Robinson SM, Reginster JY, Rizzoli R, Shaw SC, Kanis JA, Bautmans I, et al. Does nutrition play a role in the prevention and management of sarcopenia? Clin Nutr. 2018;37:1121-32.

23. Denison HJ, Cooper C, Sayer AA, Robinson SM. Prevention and optimal management of sarcopenia: a review of combined exercise and nutrition interventions to improve muscle outcomes in older people. Clin Interv Aging. 2015;10:859-69.

24. Liao CD, Chen HC, Huang SW, Liou TH. The role of muscle mass gain following protein supplementation plus exercise therapy in older adults with sarcopenia and frailty risks: a systematic review and meta-regression analysis of randomized trials. Nutrients. 2019;11:1713.

25. Kirk B, Mooney K, Cousins R, Angell P, Jackson M, Pugh JN, et al. Effects of exercise and whey protein on muscle mass, fat mass, myoelectrical muscle fatigue and health-related quality of life in older adults: a secondary analysis of the liverpool hope university-sarcopenia ageing trial (LHUSAT). Eur J Appl Physiol. 2020;120:493-503.

26. Kirk B, Mooney K, Amirabdollahian F, Khaiyat O. Exercise and dietary-protein as a countermeasure to skeletal muscle weakness: liverpool hope university - sarcopenia aging trial (LHU-SAT). Front Physiol. 2019;10:445. 
27. Nilsson MI, Mikhail A, Lan L, Di Carlo A, Hamilton B, Barnard K, et al. A five-ingredient nutritional supplement and home-based resistance exercise improve lean mass and strength in free-living elderly. Nutrients. 2020;12:2391.

28. Formica MB, Gianoudis J, Nowson CA, O'Connell SL, Milte C, Ellis KA, et al. Effect of lean red meat combined with a multicomponent exercise program on muscle and cognitive function in older adults: a 6-month randomized controlled trial. Am J Clin Nutr. 2020;112:113-28.

29. Edholm P, Strandberg E, Kadi F. Lower limb explosive strength capacity in elderly women: effects of resistance training and healthy diet. J Appl Physiol (1985). 2017;123:190-6.

30. Bunout D, Barrera G, Leiva L, Gattas V, De la Maza MP, Avendaño M, et al. Effects of vitamin D supplementation and exercise training on physical performance in Chilean vitamin D deficient elderly subjects. Exp Gerontol. 2006;41:746-52.

31. Cornish SM, Myrie SB, Bugera EM, Chase JE, Turczyn D, Pinder M. Omega-3 supplementation with resistance training does not improve body composition or lower biomarkers of inflammation more so than resistance training alone in older men. Nutr Res. 2018;60:87-95.

32. Stout JR, Smith-Ryan AE, Fukuda DH, Kendall KL, Moon JR, Hoffman JR, et al. Effect of calcium $\beta$-hydroxy- $\beta$-methylbutyrate (CaHMB) with and without resistance training in men and women 65 + yrs: a randomized, double-blind pilot trial. Exp Gerontol. 2013;48:1303-10.

33. Englund DA, Kirn DR, Koochek A, Zhu H, Travison TG, Reid KF, et al. Nutritional supplementation with physical activity improves muscle composition in mobility-limited older adults, the VIVE2 study: a randomized, double-blind, placebo-controlled trial. J Gerontol A Biol Sci Med Sci. 2018;73:95-101.

34. Fielding RA, Travison TG, Kirn DR, Koochek A, Reid KF, Von Berens $\AA$, et al. Effect of structured physical activity and nutritional supplementation on physical function in mobility-limited older adults: results from the VIVE2 randomized trial. J Nutr Health Aging. 2017;21:936-42.

35. Aguiar AF, Januário RS, Junior RP, Gerage AM, Pina FL, Do Nascimento MA, et al. Long-term creatine supplementation improves muscular performance during resistance training in older women. Eur J Appl Physiol. 2012;113:987-96.

36. Brose A, Parise G, Tarnopolsky MA. Creatine supplementation enhances isometric strength and body composition improvements following strength exercise training in older adults. J Gerontol A Biol Sci Med Sci. 2003;58:11-9.

37. Tarnopolsky M, Zimmer A, Paikin J, Safdar A, Aboud A, Pearce E, et al. Creatine monohydrate and conjugated linoleic acid improve strength and body composition following resistance exercise in older adults. PLoS One. 2007;2:e991.

38. Chilibeck PD, Kaviani M, Candow DG, Zello GA. Effect of creatine supplementation during resistance training on lean tissue mass and muscular strength in older adults: a meta-analysis. Open Access J Sports Med. 2017;8:213-26.

39. Stares A, Bains $M$. The additive effects of creatine supplementation and exercise training in an aging population: a systematic review of randomized controlled trials. J Geriatr Phys Ther. 2020;43:99-112.

40. Devries MC, Phillips SM. Creatine supplementation during resistance training in older adults-a meta-analysis. Med Sci Sports Exerc. 2014;46:1194203.

41. McKendry J, Currier BS, Lim C, McLeod JC, Thomas ACQ, Phillips SM. Nutritional supplements to support resistance exercise in countering the sarcopenia of aging. Nutrients. 2020;12:2057.

42. Zanou N, Gailly P. Skeletal muscle hypertrophy and regeneration: interplay between the myogenic regulatory factors (MRFs) and insulin-like growth factors (IGFs) pathways. Cell Mol Life Sci. 2013;70:4117-4130.

43. Morton RW, Murphy KT, McKellar SR, Schoenfeld BJ, Henselmans M, Helms E, et al. A systematic review, meta-analysis and meta-regression of the effect of protein supplementation on resistance training-induced gains in muscle mass and strength in healthy adults. Br J Sports Med. 2018;52:37684.

44. Holwerda AM, Overkamp M, Paulussen KJM, Smeets JSJ, Van Kranenburg J, Backx EMP, et al. Protein supplementation after exercise and before sleep does not further augment muscle mass and strength gains during resistance exercise training in active older men. J Nutr. 2018;148:1723-32.

45. Leenders M, Verdijk LB, Van der Hoeven L, Van Kranenburg J, Nilwik R, Wodzig WK, et al. Protein supplementation during resistance-type exercise training in the elderly. Med Sci Sports Exerc. 2013;45:542-52.

46. Mori $\mathrm{H}$, Tokuda Y. Effect of whey protein supplementation after resistance exercise on the muscle mass and physical function of healthy older women: a randomized controlled trial. Geriatr Gerontol Int. 2018;18:1398-404.

47. Nakayama K, Saito Y, Sanbongi C, Murata K, Urashima T. Effects of low-dose milk protein supplementation following low-to-moderate intensity exercise training on muscle mass in healthy older adults: a randomized placebo-controlled trial. Eur J Nutr. 2021;60:917-28.

48. Arnarson A, Gudny Geirsdottir O, Ramel A, Briem K, Jonsson PV, Thorsdottir I. Effects of whey proteins and carbohydrates on the efficacy of resistance training in elderly people: double blind, randomised controlled trial. Eur J Clin Nutr. 2013;67:821-6.

49. Nabuco HCG, Tomeleri CM, Sugihara Junior P, Fernandes RR, Cavalcante EF, Antunes M, et al. Effects of whey protein supplementation pre- or postresistance training on muscle mass, muscular strength, and functional capacity in pre-conditioned older women: a randomized clinical trial. Nutrients. 2018;10:563.

50. Sugihara Junior P, Ribeiro AS, Nabuco HCG, Fernandes RR, Tomeleri CM, Cunha PM, et al. Effects of whey protein supplementation associated with resistance training on muscular strength, hypertrophy, and muscle quality in preconditioned older women. Int J Sport Nutr Exerc Metab. 2018;28:52835.

51. O'Bryan KR, Doering TM, Morton RW, Coffey VG, Phillips SM, Cox GR. Do multi-ingredient protein supplements augment resistance training-induced gains in skeletal muscle mass and strength? A systematic review and meta-analysis of 35 trials. Br J Sports Med. 2020;54:573-81. 
52. Yannakoulia M, Ntanasi E, Anastasiou CA, Scarmeas N. Frailty and nutrition: from epidemiological and clinical evidence to potential mechanisms. Metabolism. 2016;68:64-76.

53. McLean RR, Mangano KM, Hannan MT, Kiel DP, Sahni S. Dietary protein intake is protective against loss of grip strength among older adults in the Framingham offspring cohort. J Gerontol A Biol Sci Med Sci. 2016;71:356-61.

54. Morley JE, Vellas B, Van Kan GA, Anker SD, Bauer JM, Bernabei R, et al. Frailty consensus: a call to action. J Am Med Dir Assoc. 2013;14:392-7.

55. Cruz-Jentoft AJ, Kiesswetter E, Drey M, Sieber CC. Nutrition, frailty, and sarcopenia. Aging Clin Exp Res. 2017;29:43-8.

\section{Supplementary}

Due to technical limitations, Figure 3 is only available as a download in the Supplemental Files section.

\section{Figures}

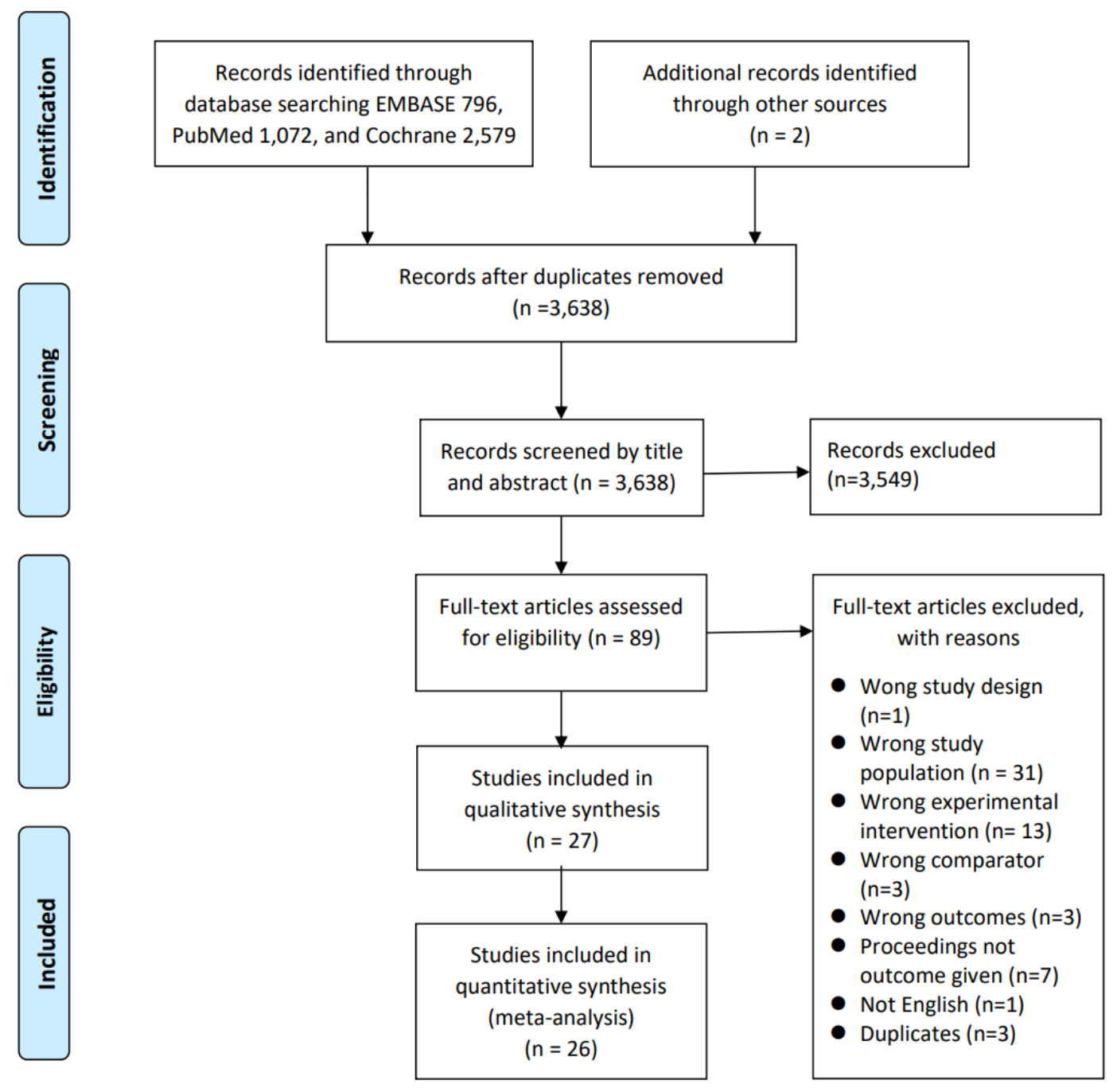

Figure 1

Flow diagram of the study selection process 


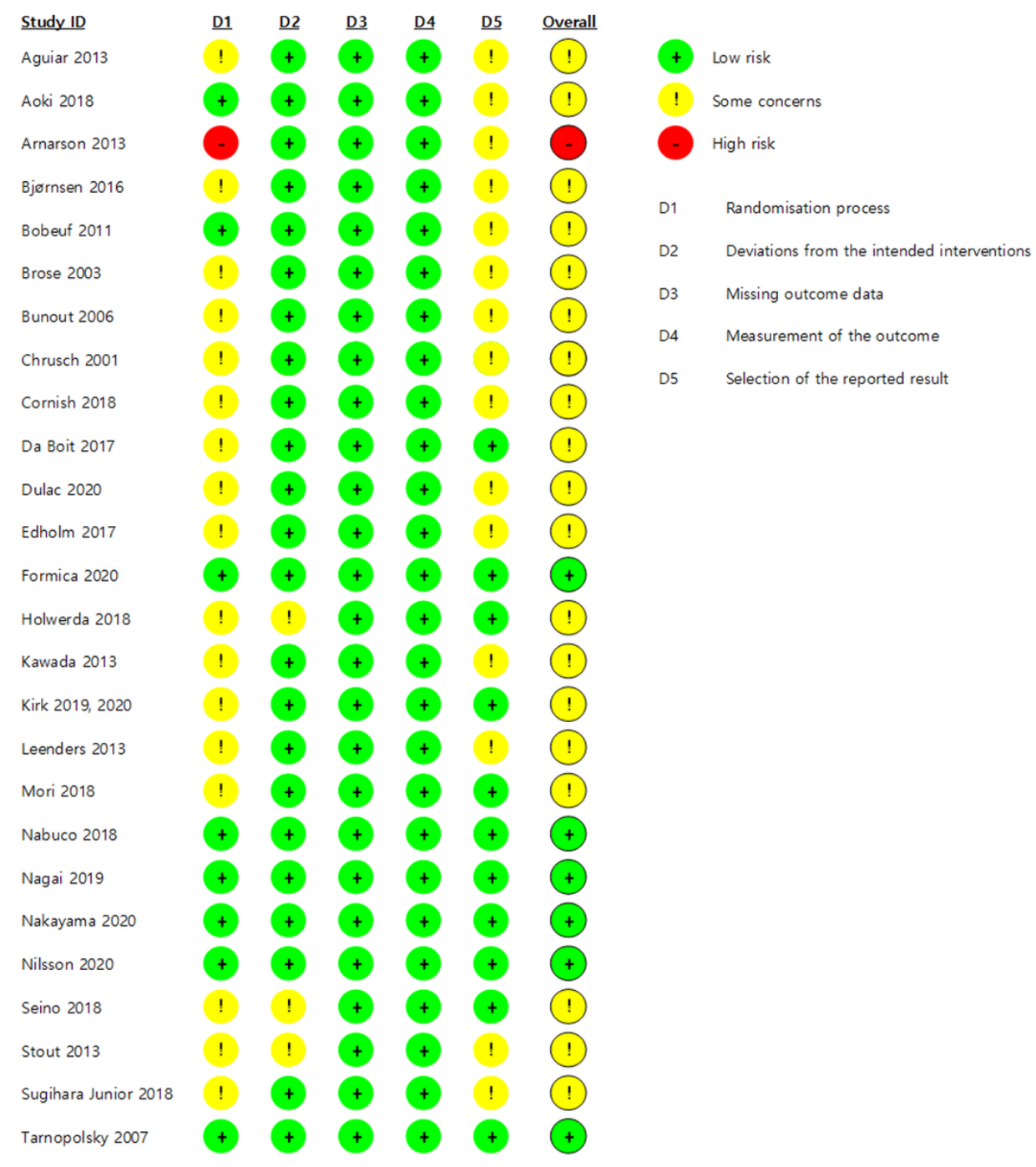

Figure 2

Risk of bias of the included studies

\section{Supplementary Files}

This is a list of supplementary files associated with this preprint. Click to download.

- Additionalfile.PRISMA2020checklist.docx

- Figure3Effectofresistancetrainingandnutritionalinterventiononmusclemassstrengthandphysicalfunctionalperformance.docx 